\title{
An integrative microenvironment approach for follicular lymphoma: roles of inflammatory cell subsets and immune- response polymorphisms on disease clinical course
}

\author{
Guilherme Rossi Assis-Mendonça ${ }^{1,2}$, André Fattori ${ }^{3}$, Rafael Malagoli Rocha ${ }^{4}$, Gustavo \\ Jacob Lourenço ${ }^{5}$, Márcia Torresan Delamain ${ }^{6}$, Suely Nonogaki ${ }^{7}$, Vladmir Cláudio \\ Cordeiro de Lima ${ }^{8}$, Gisele Wally Braga Colleoni ${ }^{9}$, Cármino Antonio de Souza ${ }^{3,6}$, \\ Fernando Augusto Soares ${ }^{10}$, Carmen Silvia Passos Lima ${ }^{3,5}$ and José Vassallo ${ }^{1,2,10}$ \\ ${ }^{1}$ Department of Pathology, Faculty of Medical Sciences, University of Campinas, Campinas, São Paulo, Brazil \\ ${ }^{2}$ Laboratory of Investigative and Molecular Pathology, Faculty of Medical Sciences, University of Campinas, Campinas, São \\ Paulo, Brazil \\ ${ }^{3}$ Department of Internal Medicine, Faculty of Medical Sciences, University of Campinas, Campinas, São Paulo, Brazil \\ ${ }^{4}$ Molecular Gynecology Laboratory, Department of Gynecology, Federal University of São Paulo, São Paulo, Brazil \\ ${ }^{5}$ Laboratory of Cancer Genetics, Faculty of Medical Sciences, University of Campinas, Campinas, São Paulo, Brazil \\ ${ }^{6}$ Hematology and Hemotherapy Center, University of Campinas, Campinas, São Paulo, Brazil \\ ${ }^{7}$ Instituto Adolfo Lutz, Secretaria de Estado da Saúde, São Paulo, Brazil \\ ${ }^{8}$ Department of Medical Oncology, AC Camargo Cancer Center, São Paulo, Brazil \\ ${ }^{9}$ Federal University of São Paulo, São Paulo, Brazil \\ ${ }^{10}$ Rede D'Or Hospitals-Pathology Department, São Paulo, Brazil \\ Correspondence to: Guilherme Rossi Assis-Mendonça, email: guilhermeram13@yahoo.com.br \\ Keywords: follicular lymphoma; tumor microenvironment; immunohistochemistry; single nucleotide polymorphisms; prognosis \\ Received: March 11, $2020 \quad$ Accepted: July 14, $2020 \quad$ Published: August 18, 2020
}

Copyright: Assis-Mendonça et al. This is an open-access article distributed under the terms of the Creative Commons Attribution License 3.0 (CC BY 3.0), which permits unrestricted use, distribution, and reproduction in any medium, provided the original author and source are credited.

\section{ABSTRACT}

The study of the tumor microenvironment (TME) in follicular lymphoma (FL) has produced conflicting results due to assessment of limited TME subpopulations, and because of heterogeneous treatments among different cohorts. Also, important genetic determinants of immune response, such as single-nucleotide polymorphisms (SNPs), remain underexplored in this disease.

We performed a detailed study of the TME in 169 FL biopsies using immunohistochemistry, encompassing lymphocytes, macrophages, and cytokines. We also genotyped 16 SNPs within key immune-response genes (IL12A, IL2, IL10, TGFB1, TGFBR1, TGFBR2, IL17A, and IL17F) in 159 patients. We tested associations between SNPs, clinicopathological features and TME composition, and proposed survival models in R-CHOP/R-CVP-treated patients.

Presence of the IL12A rs568408 "A" allele associated with the follicular pattern of FOXP3+ cells. The IL12A AA haplotype included rs583911 and rs568408 and was an independent predictor of worse survival, together with the follicular patterns of T-cells (FOXP3+ and CD8+) and high IL-17F tumor levels. The patterns of CD3+, CD4+ and CD8+ cells, displayed as a principal component, also associated with survival. Hierarchical clustering of the TME proteins demonstrated a cluster that was associated with worse prognosis (tumors enriched in IL-17A, IL-17F, CD8, PD1, and Ki-67).

The survival of FL patients who were treated in the rituximab era shows a strong dependence on TME signals, especially the T-cell infiltration patterns and IL-17F tumor levels. The presence of the AA haplotype of IL12A in the genome of FL patients is an additional prognostic factor that may modulate the composition of T-reg cells in this disease. 


\section{INTRODUCTION}

Follicular lymphoma (FL) is the most common low-grade non-Hodgkin lymphoma (NHL) subtype and is characterized by an indolent clinical course and frequent relapses [1]. The progression of FL is influenced by stromal signals [2]. In this setting, the study of the tumor microenvironment (TME), which is composed of nonneoplastic cells and related molecules, has provided important insights on the signaling mechanisms and prognosis of FL. Several studies have described the role of tumor-infiltrating lymphocytes (TILs), macrophages, and inflammatory cytokines in the modulation of the clinical course of FL [3-9]. However, these results are often non-reproducible, due to differences in the cell quantification methods (e.g., visual estimation, manual counting, automated counting) and also due to the variation in treatment patterns $[10,11]$.

Genes that encode inflammatory molecules, such as cytokines, also interfere in lymphoma biology, due to their intrinsic role in the development of lymphocytes [12]. In this setting, genomic variations, such as single-nucleotide polymorphisms (SNPs), especially variants that are associated with the modulation of immune functions, are relevant for the development and progression of lymphomas [12-14]. For instance, SNPs in key inflammatory genes, such as IL10 and $I L 2$, are more consistently studied in large NHL cohorts and have been implicated in disease risk or in prognosis in the pre-rituximab era, including certain FL cohorts $[12,13]$. Cytokines that are encoded by other immune response genes, such as IL12A,TGFB1,IL17A, and IL17F, also regulate the balance of T-cell subsets and T-cell exhaustion in B-cell lymphomas. Nevertheless, the respective SNPs have been examined in few studies and in a non-integrated fashion with other components of the TME [14-19].

No study has yet evaluated the function of SNPs within immune genes in the TME composition of FL. This is biologically plausible, especially in the case of functional variants. In addition, there is a need for more specific prognostic factors in FL. The efficacy of rituximab (anti-CD20), which is associated with better treatment outcomes, may be influenced by components of the TME $[9,10]$. Therefore a more detailed investigation of the microenvironment in patients who receive anti-CD20 is warranted.

This study aimed to verify whether SNPs in immune response genes modify the TME composition and clinical features of FL. Another goal was to test the prognostic impact of these SNPs and the TME components in a cohort of patients who have been treated with rituximabcontaining regimens.

\section{RESULTS}

\section{Clinicopathological features}

A total of $237 \mathrm{FL}$ patients were obtained (117 from UNICAMP and 120 from A. C. Camargo Cancer Center).
The median age at diagnosis was of 57 years old (range: 19-94). As expected, there was a female predominance $(133 / 237$ or $56.1 \%)$, and most of the patients (189/237 or 79.7\%) were diagnosed in advanced Ann Arbor stages (III or IV). The most common first-line treatment regimens employed were R-CHOP (107 patients) and R-CVP (65 patients). These and other characteristics are listed in Table 1.

\section{Immunohistochemical assessment of cell components of the TME}

Immunohistochemical (IHC) analysis of the TME components was possible in samples from 169/237 patients $(71.3 \%)$, which were provided as a tissue microarray (TMA). The most frequent subpopulation was T-lymphocytes (median $=21.37 \%$ of pixels), with a predominance of cells expressing CD4 (median $=18.97 \%)$ over CD8 (median $=6.09 \%$ ). The least frequent subpopulation was perforin-positive cells (median $=0.28 \%$ of pixels). Regarding the expression of cytokines, IL-17A had the strongest staining score (median $\mathrm{H}$-score $=209.84$ ), and IL-12A, the weakest (median H-score $=26.47$ ). The antibody against TGFBR2 did not pass the quality control check in the tumor samples due to non-specific staining and thus, was not further analyzed. Figure 1 illustrates representative IHC stains before and after the pixel count.

The qualitative TME assessment included an evaluation of the meshworks of follicular dendritic cells (FDCs) and the infiltration patterns of TILs. Thirty-three patients (33/147 or $22.4 \%$ ) were categorized as having no detectable FDCs (Group 1). Most of the tumors (64/147 or $43.6 \%$ ) were classified as having poorly developed FDC meshworks (Group 2), and 34/147 patients (23.1\%) were included in Group 3, which comprised predominantly welldeveloped meshworks. Finally, 16/147 patients (10.9\%) had uniformly well-developed meshworks (Group 4) (representative IHC images are in Supplementary Figure 1).

An assessment of the patterns of the TILs revealed that the $\mathrm{CD} 3+, \mathrm{CD} 4+, \mathrm{CD} 8+$ and $\mathrm{FOXP} 3+$ cells presented a non-follicular infiltration pattern in most evaluable cases $(89.3 \%, 87.2 \%, 90.2 \%$ and $69.6 \%$, respectively). In contrast, for PD1+ and CD57+ cells, the follicular pattern was more prevalent $(77.9 \%$ and $60.0 \%$ of evaluable cases) (Supplementary Table 1, Supplementary Figure 2). Moreover, significant differences in cell quantities were detected between patterns. There were higher quantities of $\mathrm{CD} 3+$ and $\mathrm{CD} 8+$ cells in cases that presented with a non-follicular pattern ( $p=0.0001$ and 0.03 , respectively); whereas for PD1 and CD57, there were increased cell counts in patients with a follicular pattern ( $p=0.02$ and 0.01, respectively) (Figure 2).

The presence of a follicular pattern in the CD8+ cells was associated with bone marrow infiltration at diagnosis $(p=0.04)$. However, the patterns of the other TILs did not correlate with clinical features (Supplementary Table 2). 
Table 1: Clinicopathological features of the follicular lymphoma patients in this study

\begin{tabular}{|c|c|}
\hline Characteristic & Number of patients $(\%)$ \\
\hline \multicolumn{2}{|l|}{ B symptoms } \\
\hline Present (\%) & $82(34.6)$ \\
\hline Absent $(\%)$ & $150(63.3)$ \\
\hline Not available (\%) & $5(2.1)$ \\
\hline \multicolumn{2}{|l|}{ FLIPI index } \\
\hline High risk $(\%)$ & $73(30.8)$ \\
\hline Intermediate risk $(\%)$ & $85(35.9)$ \\
\hline Low risk (\%) & $61(25.7)$ \\
\hline Not available (\%) & $18(7.6)$ \\
\hline \multicolumn{2}{|l|}{ Bulky disease } \\
\hline Present $(\%)$ & $58(24.5)$ \\
\hline Absent (\%) & $149(62.9)$ \\
\hline Not available (\%) & $30(12.6)$ \\
\hline \multicolumn{2}{|l|}{ Bone marrow infiltration } \\
\hline Present $(\%)$ & $102(43.0)$ \\
\hline Absent (\%) & $130(54.8)$ \\
\hline Not available (\%) & $5(2.2)$ \\
\hline \multicolumn{2}{|c|}{ Extranodal disease, excluding bone marrow } \\
\hline Present $(\%)$ & $77(32.5)$ \\
\hline Absent (\%) & $145(61.2)$ \\
\hline Not available (\%) & $15(6.3)$ \\
\hline \multicolumn{2}{|l|}{ Ann Arbor stage } \\
\hline I or II $(\%)$ & $48(20.3)$ \\
\hline III ou IV (\%) & $189(79.7)$ \\
\hline \multicolumn{2}{|l|}{ Histological grade } \\
\hline $1(\%)$ & $75(31.7)$ \\
\hline $2(\%)$ & $92(38.8)$ \\
\hline $3 \mathrm{~A}(\%)$ & $50(21.1)$ \\
\hline Not available (\%) & $20(8.4)$ \\
\hline \multicolumn{2}{|l|}{ First-line treatment } \\
\hline $\mathrm{R}-\mathrm{CHOP}(\%)$ & $107(45.1)$ \\
\hline R-CVP $(\%)$ & $65(27.4)$ \\
\hline Rituximab monotherapy (\%) & $4(1.7)$ \\
\hline CHOP/CHOP like (\%) & $40(16.9)$ \\
\hline Other drugs (\%) & $8(3.4)$ \\
\hline Watch and wait (\%) & $13(5.5)$ \\
\hline
\end{tabular}

The quantifications (pixel counts) of the panmarkers (CD3 and CD68) revealed positive correlations with most other proteins. Conversely, the presence of NK cells (CD57+) correlated inversely with Ki-67, IL-12A, iNOS, and IL-10 expression. Furthermore, there was a negative correlation between the presence of CD8+ and granzyme $\mathrm{B}+$ cells, indicating that not all $\mathrm{CD} 8+$ cells expressed cytotoxic markers. Finally, IL-17F correlated negatively with IL-12A, iNOS and granzyme B. The correlation matrix is shown in Figure 3.

The quantification of the TME cells had no impact on the clinical characteristics (data not shown). Regarding the expression of cytokines, higher IL-10 tumor levels were associated with a lower frequency of extranodal disease at diagnosis $(p=0.03)$. We also observed a trend of an association of high IL-10 levels with B-symptoms 
$(p=0.06)$ and with high-risk Follicular Lymphoma International Prognostic Index (FLIPI) $(p=0.05)$. The remaining cytokines were not associated with the clinical characteristics (Table 2).

\section{Hierarchical clustering and principal component analysis of the TME components}

To evaluate the TME proteins using a more realistic approach, we performed unsupervised hierarchical clustering of the immunohistochemical markers that were quantified using the Aperio system. Two clusters (1 and 2) resulted, with 100 and 32 patients, respectively (Figure 4A). Cluster 1 was enriched in cells that expressed CD8, perforin, CD57, PD1, and FOXP3 and exhibited higher Ki-67 counts than cluster 2. There was also higher expression of IL17A, IL-17F and TGFBR1 for cluster 1 . In contrast, cluster 2 had higher levels of granzyme B, IL-10, IL-12A, and iNOS (Figure 4B and 4C). No differences in macrophage infiltration (CD68, CD163) were seen (data not shown).

The clinical features of clusters 1 and 2 were similar with respect to the FLIPI index, B-symptoms, and bone marrow infiltration rates. The distributions of gender and age were similar as well. However, cluster 1 had a higher frequency of extranodal disease $(p=0.02)$ (Table 3$)$.

Additionally, we performed an exploratory principal component analysis (PCA) using the IHC variables (both categorical and numerical data) to complement the strictly numerical data in the clusters. The final PCA model comprised $73.01 \%$ of the variance and was composed of
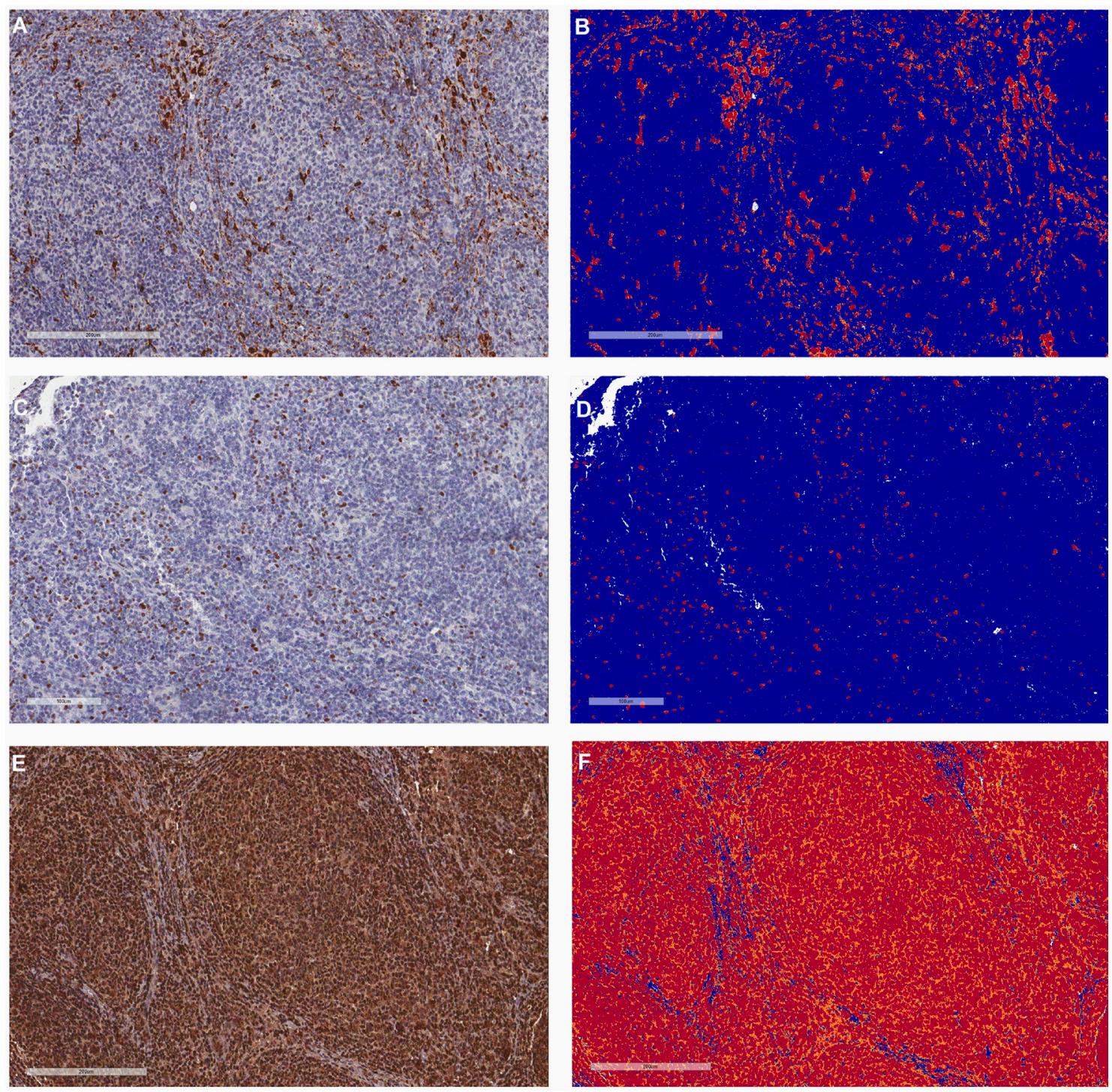

Figure 1: Representative pictures of the tumor microenvironment assessments by immunohistochemistry in follicular lymphoma. (A) and (B) Cytoplasmic staining of macrophages with anti-CD68 before and after pixel counting, respectively. (C) and (D) Nuclear staining of T-regulatory lymphocytes with anti-FOXP3 before and after pixel counting, respectively. (E) and (F) Diffuse cytoplasmic staining of IL-17F (high-expressor case) before and after pixel counting, respectively. The pixels were categorized as negative (blue), weak positive (yellow), positive (orange) and strong positive (red) by the Aperio system, following predetermined settings. 
Table 2: Intratumoral levels of cytokines and clinicopathological features of follicular lymphoma patients

\begin{tabular}{|c|c|c|c|c|c|c|c|}
\hline & IL-12A high & IL-2 high & IL-10 high & TGF $\beta$ high & TGFBR1 high & IL-17A high & IL-17F high \\
\hline \multicolumn{8}{|l|}{ B symptoms } \\
\hline Present (\%) & $28 / 53(52.8)$ & $30 / 54(55.5)$ & $34 / 54(62.9)$ & $28 / 53(52.8)$ & $31 / 52(59.6)$ & $26 / 54(48.1)$ & $30 / 54(55.5)$ \\
\hline Absent (\%) & $55 / 110(50.0)$ & $53 / 109(48.6)$ & 49/109 (44.9) & $53 / 106(50.0)$ & 48/103 (46.6) & $55 / 108(50.9)$ & $52 / 108(48.1)$ \\
\hline$p$ & 0.97 & 0.53 & 0.06 & 1.00 & 0.48 & 0.73 & 1.00 \\
\hline \multicolumn{8}{|l|}{ FLIPI } \\
\hline High risk (\%) & $22 / 45(48.9)$ & $21 / 46(45.6)$ & $27 / 46(58.7)$ & $21 / 44(47.7)$ & $23 / 42(54.7)$ & $26 / 46(56.5)$ & $26 / 46(56.5)$ \\
\hline Intermediate/low risk (\%) & $46 / 100(46.0)$ & $54 / 99(54.5)$ & 40/99 (40.4) & $48 / 98(49.0)$ & $54 / 96(56.2)$ & 49/98 (50.0) & $52 / 98(53.0)$ \\
\hline$p$ & 0.74 & 1.00 & 0.05 & 0.89 & 0.87 & 0.61 & 0.74 \\
\hline \multicolumn{8}{|l|}{$\begin{array}{l}\text { Extranodal disease } \\
\text { (excluding bone marrow) }\end{array}$} \\
\hline Present (\%) & $21 / 50(42.0)$ & $22 / 47(46.8)$ & $17 / 49(34.7)$ & $22 / 47(46.8)$ & $22 / 46(47.8)$ & $27 / 49(55.1)$ & $27 / 48(56.2)$ \\
\hline Absent (\%) & $56 / 102(54.9)$ & $55 / 105(52.3)$ & $59 / 103(57.3)$ & $53 / 101(52.4)$ & $53 / 98(54.0)$ & $48 / 102(47.0)$ & $50 / 103(48.5)$ \\
\hline$p$ & 0.52 & 0.52 & 0.03 & 1.00 & 0.96 & 1.00 & 0.37 \\
\hline \multicolumn{8}{|l|}{ Bone marrow infiltration } \\
\hline Present (\%) & $34 / 76(44.7)$ & $35 / 75(46.7)$ & $36 / 75(48.0)$ & $36 / 74(48.6)$ & $38 / 74(51.3)$ & $41 / 76(53.9)$ & $38 / 76(50.0)$ \\
\hline Absent (\%) & $48 / 87(55.1)$ & $48 / 88(54.5)$ & $46 / 88(52.2)$ & $43 / 85(50.6)$ & 39/81 (48.1) & $41 / 86(47.6)$ & $43 / 86(50.0)$ \\
\hline$p$ & 0.36 & 0.62 & 0.58 & 1.00 & 0.91 & 0.84 & 1.00 \\
\hline
\end{tabular}

All $p$-values were obtained by chi-squared test and were adjusted for multiple comparisons (Benjamini-Hochberg method). A "high" classification refers to the values above the median.

6 components (Table 4). The first component showed that elevated expression of TGFBR1, IL-12A, IL-2, and IL-10 was associated with a follicular pattern in FOXP3+ cells. The second component revealed a direct association between macrophages (CD68, CD163) and perforin expression. The third component aggregated the non-follicular proliferation patterns of CD3, CD4, and CD8 cells. The fourth component revealed an association of the follicular pattern of CD8 cells with elevated intratumoral IL-10, IL-17A, and IL-17F levels. For the fifth component, high CD68 and iNOS levels were associated with a high proliferative index (Ki-67). Finally, the sixth component demonstrated an opposing trend of high TGF $\beta$ and low IL-17A levels.

\section{SNP genotyping and associations with clinicopathological features}

A total of $159 \mathrm{FL}$ patients (71 men and 88 women) were genotyped. There was a lack of Hardy-Weinberg Equilibrium (HWE) in 4 SNPs: IL12A rs568408, IL2 rs2069762, IL10 rs3024491, and TGFBR1 rs334348 (Supplementary Table 3).

Some SNPs were predictors of the clinical features at diagnosis. The genotypes GT+TT of IL2 rs6822844, compared with the GG genotype, were predictors for bulky disease at presentation (odds ratio $[\mathrm{OR}]=2.55,95 \%$ confidence interval $[95 \% \mathrm{CI}]=1.10-5.91, p=0.029)$. In addition, the presence of the $\mathrm{CC}$ genotype for IL10 rs1800872, in comparison with the CA+AA genotypes, was associated with bone marrow infiltration $(\mathrm{OR}=2.07$, 95\% CI: 1.07-4.01, $p=0.030)$. Regarding IL10 rs1800890, the genotypes TA+AA were associated with the presence of B-symptoms when compared with the TT genotype $(\mathrm{OR}=2.58,95 \% \mathrm{CI}=1.29-5.15, p=0.007)$. Finally, the AA genotype of TGFB1 rs6957, compared with the $\mathrm{AG}+\mathrm{GG}$ genotypes, predicted the presence of extranodal disease $(\mathrm{OR}=2.17,95 \% \mathrm{CI}=1.02-4.58, p=0.042)$.

Some of the SNPs were associated with the infiltration patterns of TILs in the FL biopsies (Table 5). For instance, the follicular pattern of the FOXP3+ cells could be predicted by the GA+AA genotypes of IL12A rs568408 $(\mathrm{OR}=3.07,95 \% \mathrm{CI}=1.10-8.56, p=0.03)$. In addition, the SNP IL10 rs1800872 was associated with the follicular patterns of $\mathrm{CD} 8(\mathrm{CA}+\mathrm{AA}$ vs $\mathrm{CC}, \mathrm{OR}=8.87$, $95 \% \mathrm{CI}=1.03-76.10, p=0.04)$, PD1 (CA+AA vs CC, $\mathrm{OR}=3.59,95 \% \mathrm{CI}=1.17-11.0, p=0.02)$ and $\mathrm{CD} 57$ (AA vs $\mathrm{CC}, \mathrm{OR}=8.30,95 \% \mathrm{CI}=1.06-64.80, p=0.04)$.

Other SNPs also associated with the quantification of TME proteins. First, a higher expression of iNOS was seen in the samples from FL patients carrying the "A" allele of $I L 12 A$ rs755004 ( $p=0.005$, Figure 5A). Second, a higher percentage of granzyme $\mathrm{B}+$ cells was observed in tumors from patients with the TT genotype of $I L 17 F$ rs763780 ( $p=0.04$, Figure 5B). However, none of the SNPs altered the levels of the proteins that were encoded by each of their respective genes (data not shown).

\section{Survival analyses}

One-hundred seventy-two patients received R-CHOP or R-CVP as a first-line treatment and therefore were included in the survival analyses. The median 
Table 3: Demographic and clinical features of follicular lymphoma patients stratified by the microenvironment clusters

\begin{tabular}{lcc} 
Cluster 1 & Cluster 2 & $p$ \\
$54(54.0)$ & $15(46.9)$ & $0.54^{*}$ \\
$46(46.0)$ & $17(53.1)$ & \\
$42(42.0)$ & $13(40.7)$ & $0.89^{*}$ \\
$58(58.0)$ & $19(59.3)$ & \\
$27(28.4)$ & $9(45.0)$ & $0.14^{*}$ \\
$68(71.6)$ & $11(55.0)$ & \\
$31(31.6)$ & $10(31.2)$ & $0.96^{*}$ \\
$67(68.4)$ & $22(68.8)$ & \\
$36(37.5)$ & $4(14.2)$ & \\
$60(62.5)$ & $24(85.8)$ & \\
$47(47.0)$ & $11(35.5)$ & \\
$53(53.0)$ & $20(64.5)$ & $0.25^{*}$ \\
\hline
\end{tabular}

Age (years)

$\geq 57(\%)$

$<57(\%)$

Gender

Male (\%)

Female (\%)

$42(42.0)$

$13(40.7)$

FLIPI

High-risk (\%)

Low/intermediate risk (\%)

B symptoms

Present (\%)

$20(64.5)$

Absent (\%)

Extranodal disease (excluding bone marrow)

Present (\%)

Absent (\%)

Bone marrow infiltration

Present (\%)

Absent (\%)

The $p$ values were obtained using Chi-squared tests $\left({ }^{*}\right)$ and Fisher's exact test $\left(^{* *}\right)$.

Table 4: Principal component analysis of the tumor microenvironment in follicular lymphoma

\begin{tabular}{|c|c|c|c|c|c|c|}
\hline & \multicolumn{6}{|c|}{ Component number } \\
\hline & 1 & 2 & 3 & 4 & 5 & 6 \\
\hline TGFBR1 & 0.769 & & & & & \\
\hline KI67 & & & & & 0.87 & \\
\hline CD163 & & 0.843 & & & & \\
\hline IL-12A & 0.808 & & & & & \\
\hline Perforin & & 0.793 & & & & \\
\hline IL-10 & 0.623 & & & 0.572 & & \\
\hline TGF $\beta$ & & & & & & 0.862 \\
\hline INOS & & & & & 0.735 & \\
\hline CD68 & & 0.629 & & & 0.520 & \\
\hline Pattern- CD4 & & & 0.832 & & & \\
\hline Pattern- CD8 & & & 0.625 & -0.416 & & \\
\hline Pattern- CD3 & & & 0.868 & & & \\
\hline \multicolumn{7}{|l|}{ Pattern- CD57 } \\
\hline Pattern- FOXP3 & -0.460 & & & & & \\
\hline IL-17F & & & & 0.811 & & \\
\hline IL-17A & & & & 0.674 & & -0.576 \\
\hline IL-2 & 0.871 & & & & & \\
\hline Individual variance & $17.65 \%$ & $12.31 \%$ & $12.01 \%$ & $11.38 \%$ & $10.84 \%$ & $8.82 \%$ \\
\hline
\end{tabular}

The Italic and Bold text indicate the positive and negative values, respectively (range between -1 and +1 ). Within a single component, similar values point towards the directly associated measurements, and opposite values denote inverse associations. Considering the cell infiltration patterns, the positive values favor non-follicular patterns, and the negative numbers indicate follicular patterns. 
Table 5: Logistic regression analyses for SNPs as predictors of infiltration patterns of CD8+, PD1+, FOXP3+ and CD57+ lymphocytes

\begin{tabular}{|c|c|c|c|c|c|c|c|c|}
\hline \multirow{2}{*}{$\begin{array}{l}\text { Gene and } \\
\text { polymorphism }\end{array}$} & \multicolumn{2}{|c|}{ CD8- follicular } & \multicolumn{2}{|c|}{ PD1- follicular } & \multicolumn{2}{|c|}{ FOXP3- follicular } & \multicolumn{2}{|c|}{ CD57- follicular } \\
\hline & OR (95\% CI) & $p$ & OR $(95 \%$ CI $)$ & $p$ & OR $(95 \%$ CI $)$ & $p$ & OR $(95 \%$ CI $)$ & $p$ \\
\hline \multicolumn{9}{|l|}{ IL12A rs568408 } \\
\hline GG & 1.00 (reference) & & 1.00 (reference) & & 1.00 (reference) & & 1.00 (reference) & \\
\hline $\mathrm{GA}+\mathrm{AA}$ & $3.03(0.67-13.55)$ & 0.14 & $0.73(0.24-2.24)$ & 0.59 & $3.07(1.10-8.56)$ & 0.03 & $2.66(0.68-10.39)$ & 0.15 \\
\hline $\mathrm{AA}$ & $5.05(0.39-64.73)$ & 0.21 & $5^{8}(0-\infty)$ & 0.99 & $7.52(0.69-81.54)$ & 0.09 & $1.51(0.14-15.75)$ & 0.72 \\
\hline $\mathrm{GG}+\mathrm{GA}$ & 1.00 (reference) & & 1.00 (reference) & & 1.00 (reference) & & 1.00 (reference) & \\
\hline $\mathrm{AA}$ & $3.40(0.30-38.73)$ & 0.32 & $5^{8}(0-\infty)$ & 0.99 & $5.88(0.56-61.65)$ & 0.13 & $3.48(0.34-35.23)$ & 0.29 \\
\hline \multicolumn{9}{|l|}{ IL12A rs755004 } \\
\hline GG & 1.00 (reference) & & 1.00 (reference) & & 1.00 (reference) & & 1.00 (reference) & \\
\hline $\mathrm{GA}+\mathrm{AA}$ & $1.61(0.17-14.66)$ & 0.67 & $1.18(0.36-3.90)$ & 0.77 & $1.03(0.33-3.18)$ & 0.95 & $0.78(0.21-2.85)$ & 0.71 \\
\hline \multicolumn{9}{|l|}{ IL12A rs485497 } \\
\hline AA & 1.00 (reference) & & 1.00 (reference) & & 1.00 (reference) & & 1.00 (reference) & \\
\hline $\mathrm{AG}+\mathrm{GG}$ & $0.75(0.13-4.12)$ & 0.74 & $1.34(0.45-3.98)$ & 0.59 & $0.43(0.15-1.22)$ & 0.11 & $0.19(0.06-0.58)$ & 0.003 \\
\hline GG & $0.74(0.09-6.03)$ & 0.78 & $1.47(0.75-2.88)$ & 0.25 & $0.56(0.15-2.12)$ & 0.39 & $0.30(0.07-1.24)$ & 0.09 \\
\hline $\mathrm{AA}+\mathrm{AG}$ & 1.00 (reference) & & 1.00 (reference) & & 1.00 (reference) & & 1.00 (reference) & \\
\hline GG & $0.94(0.17-5.14)$ & 0.94 & $2.53(0.85-7.48)$ & 0.09 & $1.00(0.34-2.90)$ & 0.99 & $0.70(0.19-2.47)$ & 0.58 \\
\hline \multicolumn{9}{|l|}{ IL12A rs583911 } \\
\hline $\mathrm{AA}$ & 1.00 (reference) & & 1.00 (reference) & & 1.00 (reference) & & 1.00 (reference) & \\
\hline $\mathrm{AG}+\mathrm{GG}$ & $2.56(0.28-22.70)$ & 0.39 & $3.46(1.20-9.97)$ & 0.02 & $2.07(0.71-6.01)$ & 0.17 & $1.88(0.60-5.88)$ & 0.27 \\
\hline GG & $0.41(0.03-5.18)$ & 0.49 & $0.29(0.06-1.27)$ & 0.10 & $0.74(0.17-3.11)$ & 0.68 & $1.17(0.30-4.53)$ & 0.81 \\
\hline $\mathrm{AA}+\mathrm{AG}$ & 1.00 (reference) & & 1.00 (reference) & & 1.00 (reference) & & 1.00 (reference) & \\
\hline GG & $1.43(0.24-8.31)$ & 0.68 & $2.01(0.51-7.80)$ & 0.31 & $0.88(0.29-2.61)$ & 0.82 & $0.40(0.13-1.27)$ & 0.12 \\
\hline \multicolumn{9}{|l|}{ IL2 rs2069762 } \\
\hline TT & 1.00 (reference) & & 1.00 (reference) & & 1.00 (reference) & & 1.00 (reference) & \\
\hline $\mathrm{TG}+\mathrm{GG}$ & $1.45(0.30-7.06)$ & 0.63 & $0.58(0.21-1.60)$ & 0.30 & $1.35(0.54-3.32)$ & 0.51 & $0.65(0.24-1.80)$ & 0.41 \\
\hline \multicolumn{9}{|l|}{ IL2 $\operatorname{rs6822844}$} \\
\hline GG & 1.00 (reference) & & 1.00 (reference) & & 1.00 (reference) & & 1.00 (reference) & \\
\hline $\mathrm{GT}+\mathrm{TT}$ & $\mathrm{N} / \mathrm{E}$ & $\mathrm{N} / \mathrm{E}$ & $2.67(0.55-12.81)$ & 0.21 & $0.78(0.25-2.37)$ & 0.66 & $0.56(0.18-1.75)$ & 0.32 \\
\hline \multicolumn{9}{|l|}{ IL10 rs1800872 } \\
\hline $\mathrm{CC}$ & 1.00 (reference) & & 1.00 (reference) & & 1.00 (reference) & & 1.00 (reference) & \\
\hline $\mathrm{CA}+\mathrm{AA}$ & $8.87(1.03-76.10)$ & 0.04 & $3.59(1.17-11.0)$ & 0.02 & $2.45(0.99-6.06)$ & 0.05 & $2.43(0.87-6.77)$ & 0.08 \\
\hline $\mathrm{AA}$ & $\mathrm{N} / \mathrm{E}$ & $\mathrm{N} / \mathrm{E}$ & $6.75(1.27-35.89)$ & 0.02 & $1.58(0.32-7.63)$ & 0.56 & $8.30(1.06-64.80)$ & 0.04 \\
\hline $\mathrm{CC}+\mathrm{CA}$ & 1.00 (reference) & & 1.00 (reference) & & 1.00 (reference) & & 1.00 (reference) & \\
\hline AA & $\mathrm{N} / \mathrm{E}$ & $\mathrm{N} / \mathrm{E}$ & $3.44(0.80-14.69)$ & 0.09 & $1.02(0.22-4.58)$ & 0.97 & $5.35(0.84-33.94)$ & 0.07 \\
\hline \multicolumn{9}{|l|}{ IL10 rs3024491 } \\
\hline $\mathrm{CC}$ & 1.00 (reference) & & 1.00 (reference) & & 1.00 (reference) & & 1.00 (reference) & \\
\hline $\mathrm{CA}+\mathrm{AA}$ & $5.17(0.57-47.00)$ & 0.14 & $2.93(0.78-10.88)$ & 0.10 & $1.03(0.35-2.97)$ & 0.95 & $1.22(0.39-3.76)$ & 0.72 \\
\hline $\mathrm{AA}$ & $\mathrm{N} / \mathrm{E}$ & $\mathrm{N} / \mathrm{E}$ & $\mathrm{N} / \mathrm{E}$ & $\mathrm{N} / \mathrm{E}$ & $0.63(0.05-7.06)$ & 0.71 & $1.84(0.15-21.83)$ & 0.62 \\
\hline $\mathrm{CC}+\mathrm{CA}$ & 1.00 (reference) & & 1.00 (reference) & & 1.00 (reference) & & 1.00 (reference) & \\
\hline $\mathrm{AA}$ & $\mathrm{N} / \mathrm{E}$ & $\mathrm{N} / \mathrm{E}$ & $\mathrm{N} / \mathrm{E}$ & $\mathrm{N} / \mathrm{E}$ & $0.56(0.05-5.93)$ & 0.63 & $1.40(0.13-14.79)$ & 0.77 \\
\hline \multicolumn{9}{|l|}{ IL10 rs1800890 } \\
\hline TT & 1.00 (reference) & & 1.00 (reference) & & 1.00 (reference) & & 1.00 (reference) & \\
\hline $\mathrm{TA}+\mathrm{AA}$ & $4.25(0.72-24.91)$ & 0.10 & $2.13(0.52-8.71)$ & 0.29 & $2.44(0.83-7.16)$ & 0.10 & $1.52(0.47-4.88)$ & 0.48 \\
\hline $\mathrm{AA}$ & $\mathrm{N} / \mathrm{E}$ & $\mathrm{N} / \mathrm{E}$ & $\mathrm{N} / \mathrm{E}$ & $\mathrm{N} / \mathrm{E}$ & $1.29(0.10-16.01)$ & 0.84 & $\mathrm{~N} / \mathrm{E}$ & $\mathrm{N} / \mathrm{E}$ \\
\hline $\mathrm{TT}+\mathrm{TA}$ & 1.00 (reference) & & 1.00 (reference) & & 1.00 (reference) & & 1.00 (reference) & \\
\hline AA & $\mathrm{N} / \mathrm{E}$ & $\mathrm{N} / \mathrm{E}$ & $\mathrm{N} / \mathrm{E}$ & $\mathrm{N} / \mathrm{E}$ & $0.87(0.07-10.46)$ & 0.91 & $\mathrm{~N} / \mathrm{E}$ & $\mathrm{N} / \mathrm{E}$ \\
\hline
\end{tabular}




\section{TGFB1 rs1800469}

\begin{tabular}{|c|c|c|c|c|c|c|c|c|}
\hline $\mathrm{CC}$ & 1.00 (reference) & & 1.00 (reference) & & 1.00 (reference) & & 1.00 (reference) & \\
\hline $\mathrm{CT}+\mathrm{TT}$ & $2.17(0.37-12.61)$ & 0.38 & $1.39(0.41-4.69)$ & 0.59 & $0.81(0.27-2.36)$ & 0.70 & $0.72(0.23-2.23)$ & 0.57 \\
\hline TT & $22.8(1.15-450.48)$ & 0.04 & $0.93(0.07-11.22)$ & 0.95 & $1.78(0.20-15.43)$ & 0.60 & $0.57(0.02-11.90)$ & 0.72 \\
\hline $\mathrm{CC}+\mathrm{CT}$ & 1.00 (reference) & & 1.00 (reference) & & 1.00 (reference) & & 1.00 (reference) & \\
\hline TT & $24.38(1.62-366.71)$ & 0.02 & $0.64(0.06-6.77)$ & 0.71 & $1.95(0.25-15.27)$ & 0.52 & $0.51(0.02-9.19)$ & 0.65 \\
\hline \multicolumn{9}{|c|}{$T G F B 1$ rs1800471 } \\
\hline GG & 1.00 (reference) & & 1.00 (reference) & & 1.00 (reference) & & 1.00 (reference) & \\
\hline $\mathrm{GC}+\mathrm{CC}$ & $1.92(0.19-19.28)$ & 0.57 & $\mathrm{~N} / \mathrm{E}$ & $\mathrm{N} / \mathrm{E}$ & $2.22(0.49-10.03)$ & 0.29 & $1.21(0.21-6.90)$ & 0.82 \\
\hline \multicolumn{9}{|c|}{ TGFB1 rs6957 } \\
\hline AA & 1.00 (reference) & & 1.00 (reference) & & 1.00 (reference) & & 1.00 (reference) & \\
\hline $\mathrm{AG}+\mathrm{GG}$ & $2.18(0.45-10.47)$ & 0.33 & $1.04(0.38-2.87)$ & 0.93 & $1.50(0.61-3.66)$ & 0.37 & $1.09(0.39-3.00)$ & 0.86 \\
\hline GG & $\mathrm{N} / \mathrm{E}$ & $\mathrm{N} / \mathrm{E}$ & $0.10(0.00-1.35)$ & 0.08 & $\mathrm{~N} / \mathrm{E}$ & $\mathrm{N} / \mathrm{E}$ & $\mathrm{N} / \mathrm{E}$ & $\mathrm{N} / \mathrm{E}$ \\
\hline $\mathrm{AA}+\mathrm{AG}$ & 1.00 (reference) & & 1.00 (reference) & & 1.00 (reference) & & 1.00 (reference) & \\
\hline GG & $\mathrm{N} / \mathrm{E}$ & $\mathrm{N} / \mathrm{E}$ & $0.07(0.00-1.00)$ & 0.05 & $\mathrm{~N} / \mathrm{E}$ & $\mathrm{N} / \mathrm{E}$ & $\mathrm{N} / \mathrm{E}$ & $\mathrm{N} / \mathrm{E}$ \\
\hline \multicolumn{9}{|c|}{$T G F B R 1$ rs334348 } \\
\hline AA & 1.00 (reference) & & 1.00 (reference) & & 1.00 (reference) & & 1.00 (reference) & \\
\hline $\mathrm{AG}+\mathrm{GG}$ & $1.73(0.31-9.72)$ & 0.52 & $2.67(0.96-7.43)$ & 0.05 & $0.83(0.33-2.09)$ & 0.69 & $0.87(0.31-2.43)$ & 0.79 \\
\hline GG & $\mathrm{N} / \mathrm{E}$ & $\mathrm{N} / \mathrm{E}$ & $\mathrm{N} / \mathrm{E}$ & $\mathrm{N} / \mathrm{E}$ & $\mathrm{N} / \mathrm{E}$ & $\mathrm{N} / \mathrm{E}$ & $0.54(0.02-12.90)$ & 0.70 \\
\hline $\mathrm{AA}+\mathrm{AG}$ & 1.00 (reference) & & 1.00 (reference) & & 1.00 (reference) & & 1.00 (reference) & \\
\hline GG & $\mathrm{N} / \mathrm{E}$ & $\mathrm{N} / \mathrm{E}$ & $\mathrm{N} / \mathrm{E}$ & $\mathrm{N} / \mathrm{E}$ & $\mathrm{N} / \mathrm{E}$ & $\mathrm{N} / \mathrm{E}$ & $0.67(0.03-12.28)$ & 0.79 \\
\hline \multicolumn{9}{|c|}{$T G F B R 2$ rs3087465 } \\
\hline GG & 1.00 (reference) & & 1.00 (reference) & & 1.00 (reference) & & 1.00 (reference) & \\
\hline $\mathrm{GA}+\mathrm{AA}$ & $0.52(0.11-2.40)$ & 0.40 & $0.73(0.27-1.95)$ & 0.54 & $1.39(0.57-3.37)$ & 0.46 & $0.94(0.35-2.56)$ & 0.91 \\
\hline AA & $\mathrm{N} / \mathrm{E}$ & $\mathrm{N} / \mathrm{E}$ & $0.07(0.0-0.84)$ & 0.03 & $2.58(0.30-22.13)$ & 0.38 & $\mathrm{~N} / \mathrm{E}$ & $\mathrm{N} / \mathrm{E}$ \\
\hline $\mathrm{GG}+\mathrm{GA}$ & 1.00 (reference) & & 1.00 (reference) & & 1.00 (reference) & & 1.00 (reference) & \\
\hline AA & $\mathrm{N} / \mathrm{E}$ & $\mathrm{N} / \mathrm{E}$ & $0.07(0.0-0.76)$ & 0.03 & $2.17(0.27-17.19)$ & 0.46 & $\mathrm{~N} / \mathrm{E}$ & $\mathrm{N} / \mathrm{E}$ \\
\hline \multicolumn{9}{|c|}{ IL17A rs3748067 } \\
\hline $\mathrm{CC}$ & 1.00 (reference) & & 1.00 (reference) & & 1.00 (reference) & & 1.00 (reference) & \\
\hline $\mathrm{CT}+\mathrm{TT}$ & $0.31(0.03-2.81)$ & 0.30 & $0.50(0.18-1.41)$ & 0.19 & $0.70(0.25-1.93)$ & 0.49 & $0.59(0.20-1.70)$ & 0.33 \\
\hline \multicolumn{9}{|c|}{$I L 17 F$ rs763780 } \\
\hline TT & 1.00 (reference) & & 1.00 (reference) & & 1.00 (reference) & & 1.00 (reference) & \\
\hline $\mathrm{TC}+\mathrm{CC}$ & $0.63(0.10-3.64)$ & 0.60 & $0.40(0.14-1.15)$ & 0.09 & $0.70(0.26-1.87)$ & 0.48 & $0.63(0.20-1.92)$ & 0.41 \\
\hline
\end{tabular}

OR $(95 \% \mathrm{CI})=$ Odds ratio with $95 \%$ confidence interval. $\mathrm{N} / \mathrm{E}=$ Not evaluable $(\mathrm{OR}=$ zero or $\mathrm{OR}=\infty)$.

follow-up times were of 6.05 years (all patients) and 6.32 years (living patients). At the end of the follow-up, 136 patients were alive (79.06\%) and $36(20.94 \%)$ had died.

We first evaluated the role of the main clinical features in event-free survival (EFS) and overall survival (OS) by estimating their hazard ratios (HRs) by univariate Cox regressions. As expected, the presence of high-risk FLIPI was associated with worse EFS (HR $=1.77,95 \%$ CI: $1.08-2.88, p=0.02)$ and $\mathrm{OS}(\mathrm{HR}=3.29,95 \% \mathrm{CI}$ : $1.64-6.59, p=0.001)$ by univariate analysis. The same was observed for the presence of B-symptoms $(\mathrm{HR}=2.39$, 95\% CI: $1.49-3.86, p<0.001$ for $\mathrm{EFS}$ and $\mathrm{HR}=4.00$, 95\% CI: 2.02-7.93, $p<0.001$ for OS). The presence of extranodal disease (excluding bone marrow) was also associated with worse EFS (HR $=1.86,95 \%$ CI: 1.15 $3.01, p<0.01)$ and $\mathrm{OS}(\mathrm{HR}=2.48,95 \% \mathrm{CI}: 1.23-4.98$, $p<0.01)$. Bone marrow infiltration was marginally associated with worse OS (HR $=1.89,95 \% \mathrm{CI}: 0.96-3.74$, $p=0.06$ ), but not with EFS (HR $=1.39,95 \%$ CI: $0.87-$ $2.22, p=0.15)$. Finally, the administration of maintenance rituximab was associated with prolonged OS $(\mathrm{HR}=0.40$, 95\% CI: $0.19-0.85, p=0.01)$, but did not affect EFS $(\mathrm{HR}=0.67,95 \% \mathrm{CI}: 0.41-1.09)$.

For the univariate analysis, the presence of follicular patterns for CD8 and CD57 was associated with worse EFS ( $p=0.05$ and 0.03 , respectively); whereas, the follicular pattern of FOXP3 was associated with both worse EFS $(p=0.04)$ and OS $(p=0.01)$ (Kaplan-Meier curves on Figures $6 \mathrm{~A}-6 \mathrm{C}$ and $7 \mathrm{~A})$. In addition, high immunohistochemical expression of IL-17F and the presence of FDC meshworks (Groups 2, 3 and 4) were associated with worse EFS ( $p=0.01$ for both markers, Kaplan-Meier curves on Figure 6D and 6E). Finally, a trend toward improved EFS $(p=0.06)$ and OS $(p=0.08)$ 
was seen in cases with high number of cells that expressed granzyme B (Figures 6F and 7B). After the multivariate analysis, the immunohistochemical variables that were independently associated with worse EFS included a follicular pattern of CD8+ cells $(p=0.001)$ and elevated expression of IL-17F $(p=0.04)$. Conversely, the presence of a follicular pattern of FOXP3+ cells was the only variable that was independently associated with worse OS $(p=0.02)$ (Table 6).

The prognostic roles of all SNPs and haplotypes were also evaluated. Linkage disequilibrium (LD) analyses allowed for the construction of haplotypes in IL10 and IL12A (Figure 8; frequencies of all the haplotypes in Supplementary Table 4). The AA-genotype of the polymorphism IL10 rs1800872 was slightly associated with worse OS by univariate analysis ( $p=$ 0.06 , Figure $7 C$ ). In addition, the presence of a haplotype on IL12A (AA), involving the rs583911 and rs568408 "A" alleles, was associated with worse EFS and OS ( $p<$ 0.001 and $p=0.003$, respectively; Kaplan-Meier curves in Figures 6G and 7D). After the multivariate analysis, the AA haplotype remained significant for EFS $(p=0.01)$ and was marginally associated with OS ( $p=0.07)$ (Table 6).

Finally, we examined whether variables from the dimension-reducing analyses (PCA and clusters) altered the survival rates. In this setting, the presence of the third principal component was associated with prolonged EFS.
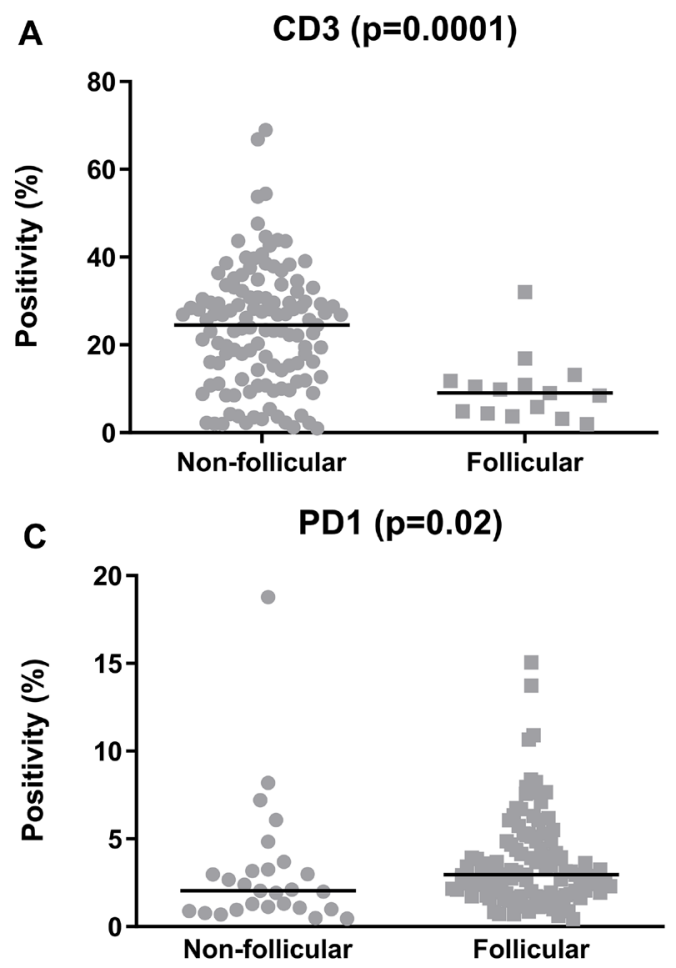

In other words, patients with non-follicular infiltration patterns for CD3, CD4 and CD8 lymphocytes had better EFS $(p=0.02)$, and the statistical significance was maintained after the multivariate analysis $(p=0.01$, Table 6). Complementarily, patients in cluster 1 had poorer EFS $(p=0.01)$ and a tendency toward worse OS $(p=0.06)$ than those in cluster 2 (Figures $6 \mathrm{H}$ and $7 \mathrm{E}$ ). After adjusting for FLIPI, cluster 1 remained an independent predictor of worse EFS ( $p=0.03)$ (Table 7).

\section{DISCUSSION}

We found that in FL patients uniformly treated with R-CHOP/R-CVP, the T-cell infiltration patterns, more importantly than quantification of these cells, independently associate with prognosis. In addition, we described a novel adverse and independent prognostic role of the AA haplotype in $I L 12 \mathrm{~A}$.

We reproduced previous successful evaluations of T-cell architectural patterns using TMAs [5, 7, 20] and found that the follicular pattern for FOXP3 + cells (T-reg cells) was independently associated with worse OS. Farinha et al. (2010) reported that this parameter shortened the time to transformation of FL in the pre-rituximab era [5]. We hereby confirm that this adverse impact also exists in patients receiving anti-CD20 therapy. In FL, FOXP3+ cells suppress the immune function of other T-cells,
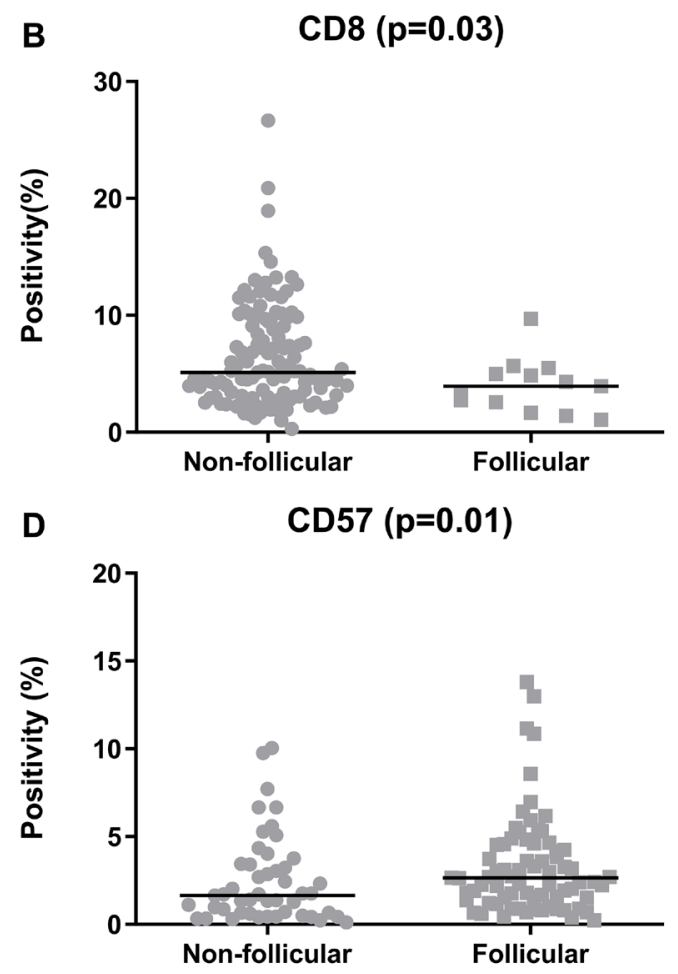

Figure 2: Quantification of the tumor-infiltrating lymphocytes in the follicular lymphoma samples, categorized according to the predominant infiltration pattern. (A) CD3, (B) CD8, (C) PD1, (D) CD57. The graphics show the median positivity levels and ranges, which were compared using two-tailed Mann-Whitney tests. The graphics for CD4 and FOXP3 were omitted, as no significance was achieved. 
Table 6: Cox regression for survival of follicular lymphoma patients

\begin{tabular}{|c|c|c|c|c|c|c|c|c|}
\hline \multirow[t]{2}{*}{ Variable } & \multicolumn{4}{|c|}{ Univariate } & \multicolumn{4}{|c|}{ Multivariate* } \\
\hline & EFS HR $(95 \% \mathrm{CI})$ & $p$ & OS HR $(95 \% \mathrm{CI})$ & $p$ & EFS HR (95\% CI) & $p$ & OS HR $(95 \% \mathrm{CI})$ & $p$ \\
\hline \multicolumn{9}{|l|}{ Immunohistochemical model } \\
\hline \multicolumn{9}{|l|}{ Pattern - CD8 } \\
\hline Follicular & $2.17(0.95-4.93)$ & 0.06 & $2.35(0.77-7.16)$ & 0.13 & $10.99(2.78-43.42)$ & 0.001 & N/A & N/A \\
\hline Non-follicular & Reference & & Reference & & Reference & & Reference & \\
\hline \multicolumn{9}{|l|}{ Pattern - FOXP3 } \\
\hline Follicular & $1.96(1.00-3.84)$ & 0.04 & $3.25(1.17-9.03)$ & 0.02 & $1.42(0.62-3.25)$ & 0.40 & $7.78(1.40-43.22)$ & 0.02 \\
\hline Non-follicular & Reference & & Reference & & Reference & & Reference & \\
\hline \multicolumn{9}{|l|}{ Pattern - CD57 } \\
\hline Follicular & $2.18(1.05-4.49)$ & 0.04 & $1.04(0.37-2.86)$ & 0.93 & $1.02(0.42-2.50)$ & 0.95 & $\mathrm{~N} / \mathrm{A}$ & N/A \\
\hline Non-follicular & Reference & & Reference & & Reference & & Reference & \\
\hline \multicolumn{9}{|l|}{ IL-17F } \\
\hline High & $2.08(1.16-3.71)$ & 0.01 & $1.98(0.82-4.78)$ & 0.12 & $2.32(1.01-5.31)$ & 0.04 & N/A & N/A \\
\hline Low & Reference & & Reference & & Reference & & Reference & \\
\hline \multicolumn{9}{|l|}{ Granzyme B } \\
\hline High & $0.57(0.32-1.03)$ & 0.06 & $0.44(0.17-1.15)$ & 0.09 & $0.67(0.28-1.58)$ & 0.36 & $0.37(0.07-1.88)$ & 0.23 \\
\hline Low & Reference & & Reference & & Reference & & Reference & \\
\hline \multicolumn{9}{|l|}{ CD23 group } \\
\hline 1 & Reference & & Reference & & Reference & & Reference & \\
\hline 2,3 or 4 & $3.55(1.26-9.95)$ & 0.01 & $31.25(0.28-3467.8)$ & 0.15 & $1.66(0.37-7.39)$ & 0.50 & $\mathrm{~N} / \mathrm{A}$ & N/A \\
\hline \multicolumn{9}{|l|}{ Genetic model } \\
\hline \multicolumn{9}{|l|}{$\begin{array}{l}\text { ILI2A AA haplotype } \\
\text { (rs583911 and rs568408) }\end{array}$} \\
\hline Present & $2.80(1.54-5.11)$ & 0.001 & $3.49(1.44-8.43)$ & 0.005 & $2.25(1.18-4.27)$ & 0.01 & $2.82(0.89-8.92)$ & 0.07 \\
\hline Absent & Reference & & Reference & & Reference & & Reference & \\
\hline \multicolumn{9}{|l|}{ rs1800872 (IL10) } \\
\hline AA & $1.28(0.50-3.24)$ & 0.59 & $2.75(0.91-8.27)$ & 0.07 & N/A & $\mathrm{N} / \mathrm{A}$ & $1.03(0.37-2.88)$ & 0.96 \\
\hline $\mathrm{AC}+\mathrm{CC}$ & Reference & & Reference & & Reference & & Reference & \\
\hline \multicolumn{9}{|l|}{ Principal components model } \\
\hline $\begin{array}{l}\text { Third microenvironment } \\
\text { component (numerical value) }\end{array}$ & $0.68(0.49-0.95)$ & 0.02 & $0.81(0.48-1.35)$ & 0.42 & $0.59(0.40-0.89)$ & 0.01 & N/A & N/A \\
\hline
\end{tabular}

EFS = event-free survival; OS = overall survival; HR = hazard ratio; 95\% CI = 95\% confidence interval; FLIPI: Follicular Lymphoma International Prognostic Index; (*) Adjustment for FLIPI, B-symptoms and extranodal disease (EFS); adjustment for FLIPI, B-symptoms, extranodal disease, bone marrow infiltration and the use of rituximab as maintenance therapy (OS).

impairing immunosurveillance [21]. Also, FL cells secrete CCL22, which induces the recruitment of T-reg cells [22]. Hence, it is conceivable that the concentration of T-reg cells near the FL cells (follicular pattern) is a histological representation of these immunosuppressive interactions.

Our findings also demonstrate that the follicular pattern of CD8+ cells was an independent factor for worse EFS, which is a novel finding for FL. In addition, one principal component encompassing non-follicular proliferations of $\mathrm{T}$ cell subsets $(\mathrm{CD} 3+, \mathrm{CD} 4+$ and $\mathrm{CD} 8+$ ), showed a favorable impact on EFS, which complements what was observed inside the neoplastic follicles. Indeed, T-cells in the interfollicular compartment seem to have preserved immune synapses and enhanced granzyme B production, possibly due to the involvement of the "border patrolling" effect $[7,23,24]$. In this setting, our results are plausible and corroborate the findings of Wahlin et al. (2010), associating non-follicular CD8+ cells with a good outcome in FL patients who have been treated in the prerituximab era [7].

Conversely, the presence of CD8+ lymphocytes in direct contact with FL cells (i.e. follicular proliferation pattern) promotes a defective immunological synapse due to the impairment of the CD8+ cell's F-actin cytoskeleton [25]. In addition, a subset of T-CD8+ cells in FL, located in the follicular compartment, may co-express molecules, such as ETV1, impairing immune function [26]. Therefore, it is plausible that CD8+ cells that are arranged in follicular patterns represent anergic subpopulations, which is reinforced by our finding associating the follicular patterns of CD8+ cells with worse outcomes.

Our initial survival analysis, as discussed above, showed that the T-cell patterns were associated with prognosis. However, none of the individual cell quantities significantly influenced survival. Notably, when we collectively evaluated these cells by hierarchical 
Table 7: Univariate and FLIPI-adjusted Cox regressions for the microenvironment clusters in follicular lymphoma patients

\begin{tabular}{|c|c|c|c|c|c|c|c|c|}
\hline & \multicolumn{4}{|c|}{ Univariate } & \multicolumn{4}{|c|}{ FLIPI-adjusted } \\
\hline & EFS HR $(95 \%$ CI $)$ & $p$ & OS HR (95\% CI) & $p$ & EFS HR $(95 \%$ CI) & $p$ & OS HR (95\% CI) & $p$ \\
\hline \multicolumn{9}{|l|}{ Cluster } \\
\hline 1 & $2.57(1.16-5.70)$ & 0.02 & $3.54(0.82-15.15)$ & 0.08 & $2.91(1.08-7.32)$ & 0.03 & $6.66(0.88-50.47)$ & 0.06 \\
\hline 2 & Reference & & Reference & & Reference & & Reference & \\
\hline \multicolumn{9}{|l|}{ FLIPI } \\
\hline High risk & $1.77(1.08-2.88)$ & 0.007 & $3.29(1.64-6.59)$ & 0.001 & $2.03(1.12-3.68)$ & 0.03 & $4.32(1.80-10.36)$ & 0.001 \\
\hline Low/intermediate risk & Reference & & Reference & & Reference & & Reference & \\
\hline
\end{tabular}

EFS = event-free survival; OS = overall survival; $\mathrm{HR}=$ hazard ratio; 95\% CI = 95\% confidence interval; FLIPI: Follicular Lymphoma International Prognostic Index.

clustering, it became evident that the patients from cluster 1 had a poorer survival, independent of the FLIPI. Cluster 1 was enriched in cells with a high proliferation index (Ki67) and in some T-cell subsets (CD8+, PD1+, FOXP3+). This cluster also presented with elevated expression of TGFBR1, IL-17A, and IL-17F. These results suggest that the quantities of the inflammatory cells, including T-cells, are important when they interact with each other and with cytokines, which reflects the complexity of TME in FL [27]. The mechanisms of these interactions should be explored further in future studies.
However, IL-17F was overexpressed in cluster 1 and was also found individually as an adverse prognostic factor for EFS. A previous report associated IL-17F expression with a bad clinical outcome of T-cell lymphomas, whereas for B-cell neoplasms, the scenario was unclear [14, 15]. In this study, we described a novel adverse prognostic role for IL-17F in FL patients, mirroring what has been observed in T-cell lymphomas. The mechanism of this association has not been described, but it may reside in the downstream effects of IL-17F, such as angiogenic sprouting and prostaglandin production [14].

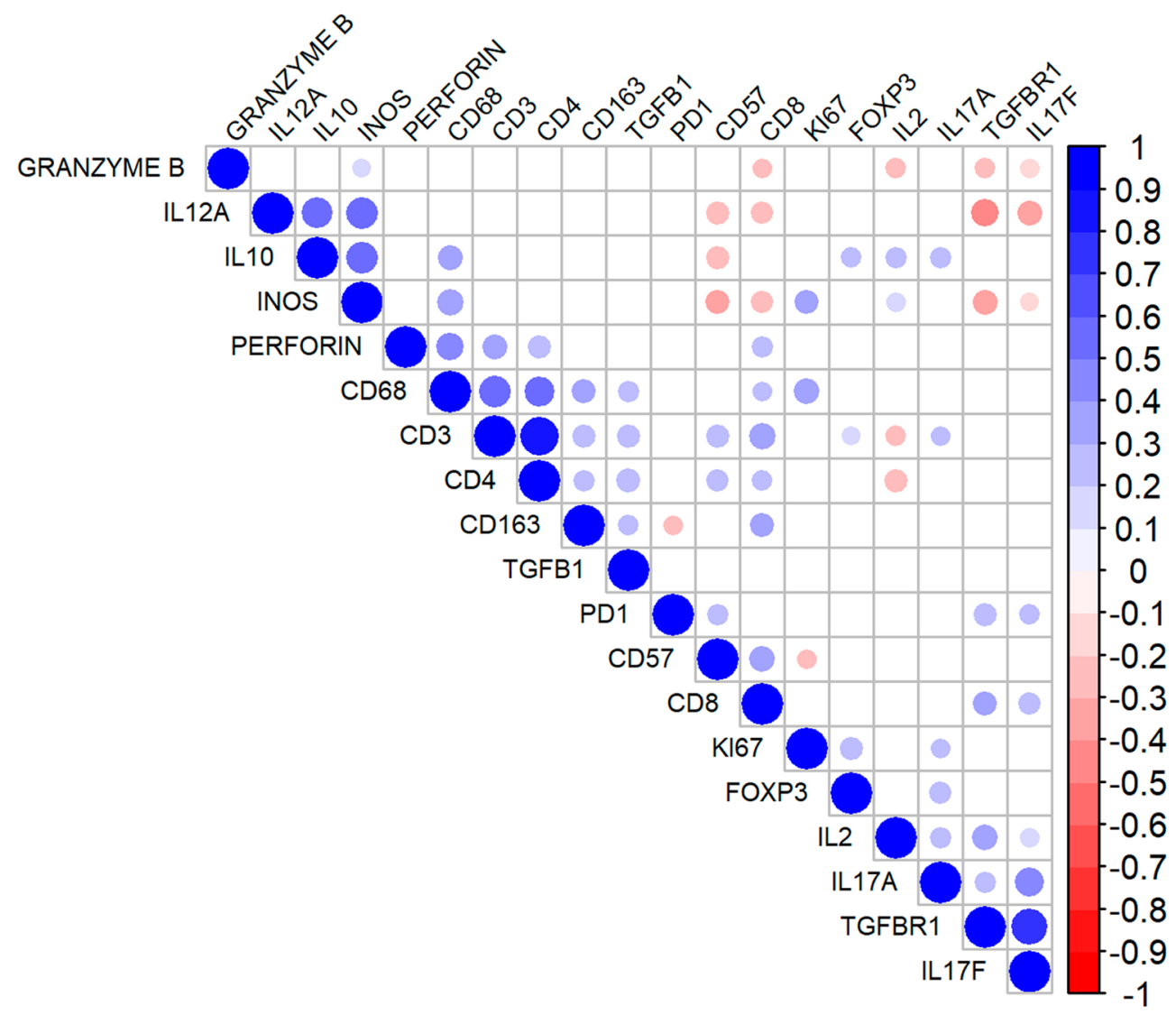

Figure 3: Correlation matrix for the immunohistochemical markers in follicular lymphoma. The diameter of the circles denotes higher modules of the correlation coefficient (r). The blueish tones indicate higher positive correlations, and the reddish tones denote stronger negative correlations. The non-significant correlations are indicated by the colorless intersections. 
A

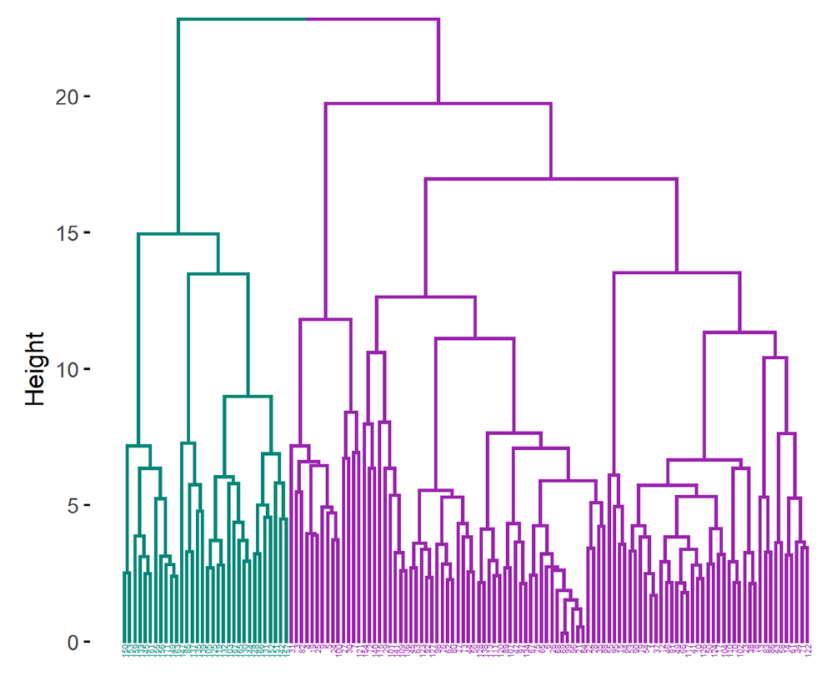

B

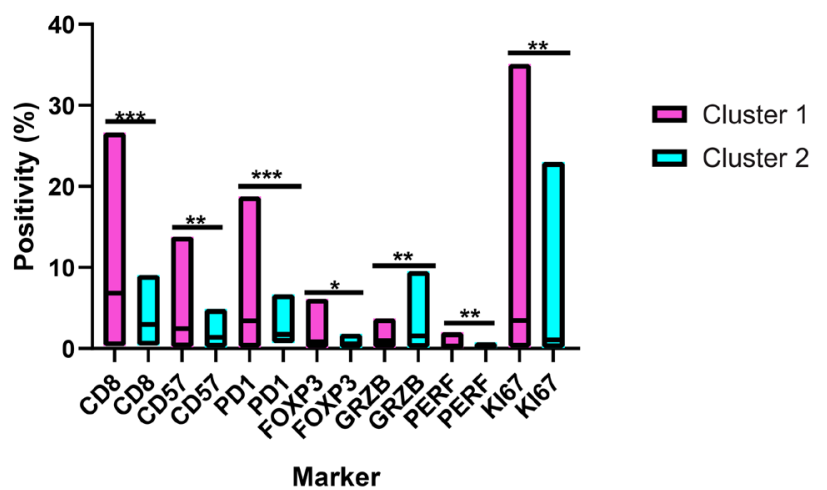

C

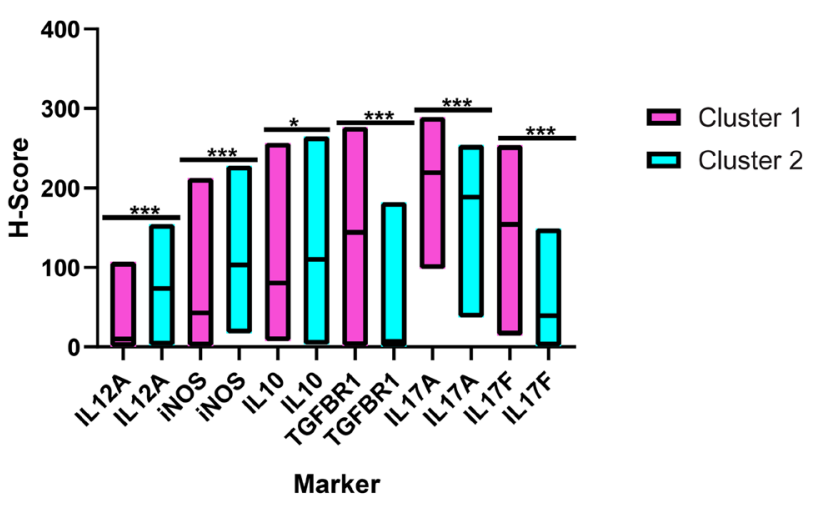

Figure 4: Hierarchical clustering of the tumor microenvironment in follicular lymphoma. (A) Dendrogram illustrating the cluster analysis of the follicular lymphoma patients based on the immunohistochemical quantifications (cluster 1 in purple and cluster 2 in blue). (B) and (C) Differential expression patterns of the tumor microenvironment cells and cytokines between clusters 1 and 2. The bars show the range of the measurements, and the horizontal lines denote the median values. ${ }^{*} p \leq 0.05 ;{ }^{* *} p \leq 0.01 ;{ }^{* * *} p \leq 0.001$ (two-tailed MannWhitney test). GRZB = Granzyme B; PERF = Perforin.
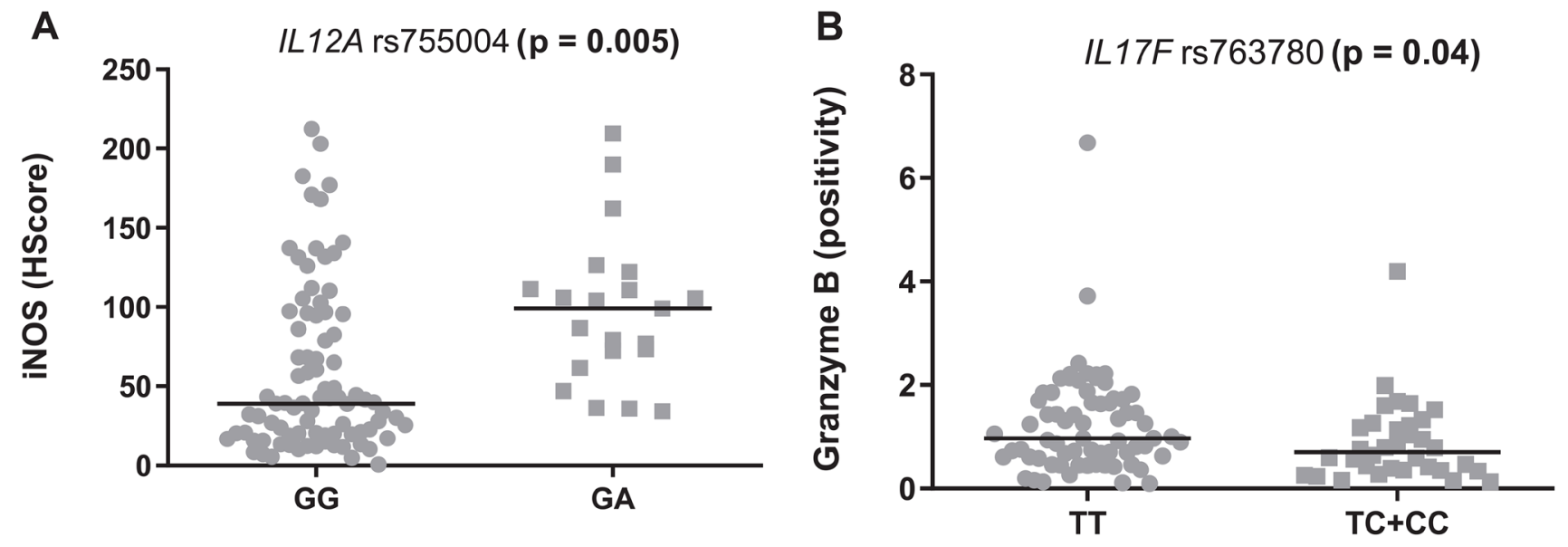

Figure 5: Modulation of the tumor microenvironment in follicular lymphoma by inflammatory SNPs. (A) rs755004 genotypes and iNOS levels. (B) rs763780 and granzyme B levels. The graphics show the median positivity levels and ranges, which were compared using two-tailed Mann-Whitney tests. The $p$ values were adjusted for multiple comparisons (Benjamini-Hochberg method). 

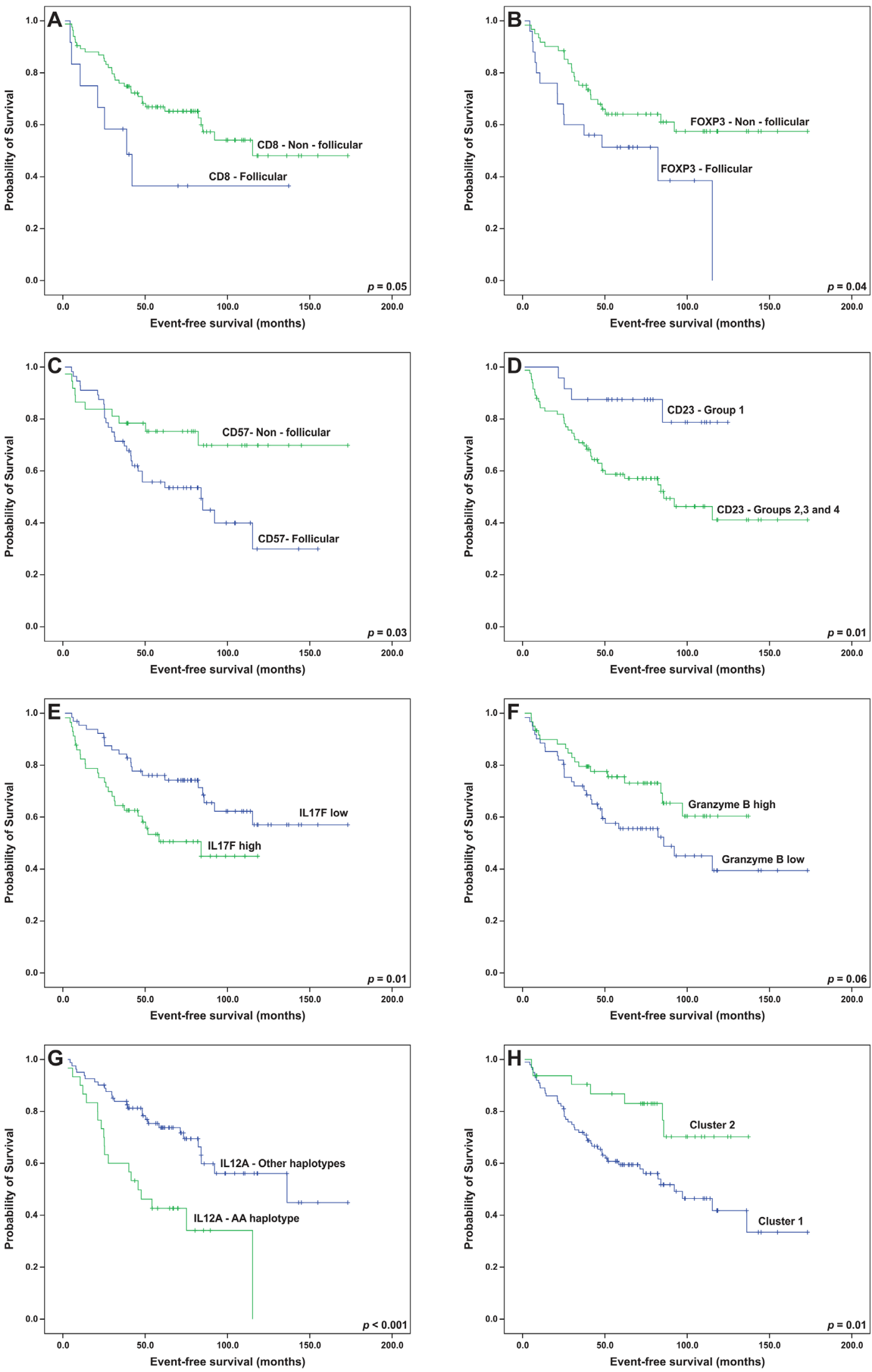

Figure 6: Event-free survival analyses (Kaplan-Meier curves) of follicular lymphoma patients treated with R-CHOP/R-CVP influenced by the patterns of (A) CD8, (B) FOXP3, (C) CD57 and (D) CD23, (E) intratumoral levels of IL-17F, (F) intratumoral levels of granzyme $\mathrm{B},(\mathbf{G})$ the presence of the $I L 12 \mathrm{~A}$ AA haplotype and $(\mathbf{H})$ microenvironment clusters. All the $p$-values were obtained from log-rank tests. 
To complement the TME investigation in FL, we examined the genetics of the host. The AA haplotype of IL12A (constituted by rs583911 and rs568408 "A" alleles) was independently associated with worse EFS and was marginally associated with OS. Two previous studies did not find any association between rs568408 and outcome in FL. However, the cohorts in these studies were predominantly from the pre-rituximab era, limiting a direct comparison with our results $[13,28]$. The explanation for the prognostic role of the AA haplotype may reside in the
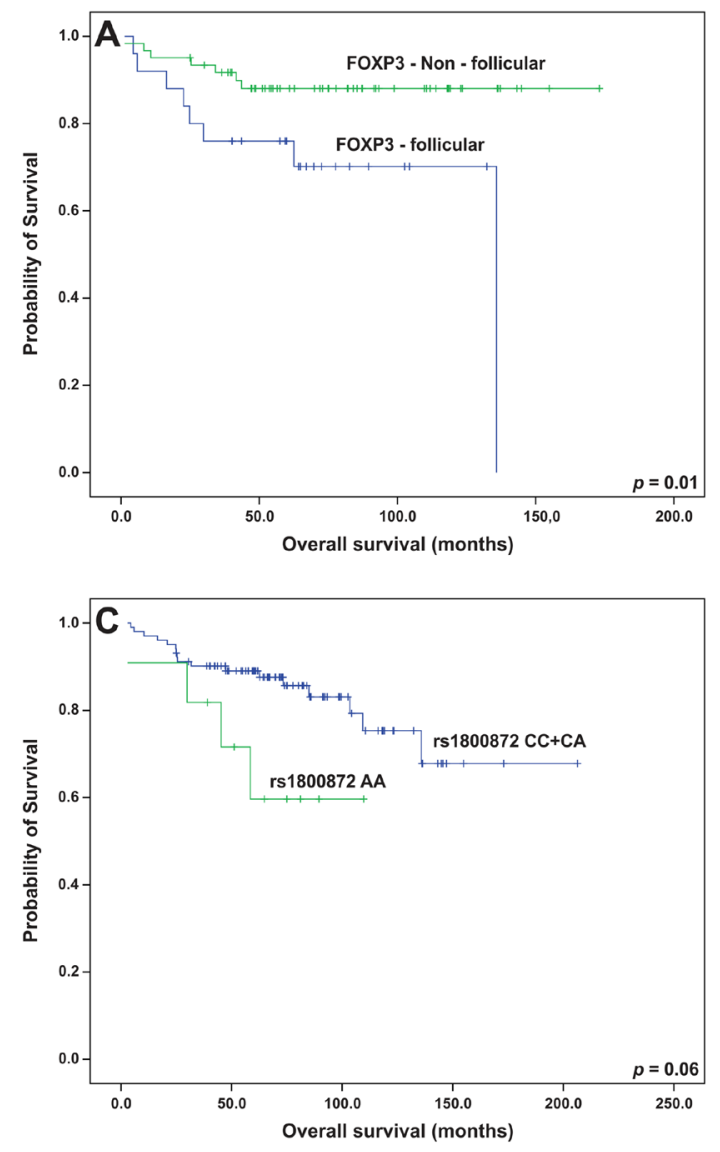

association between the "A" allele of rs568408 and the follicular pattern of the distribution of the FOXP3+ cells. However, the exact mechanism of this association has not been elucidated. It is known that the rs568408 "A" allele may lower the activity of IL-12A by disrupting exonic splicing sites [29]. In this setting, a microenvironment with less active IL-12A may favor the outgrowth of fully immunosuppressive T-reg cells, which in FL, seems to be represented by such an arrangement as a follicular pattern, according to our results and others $[5,30]$.
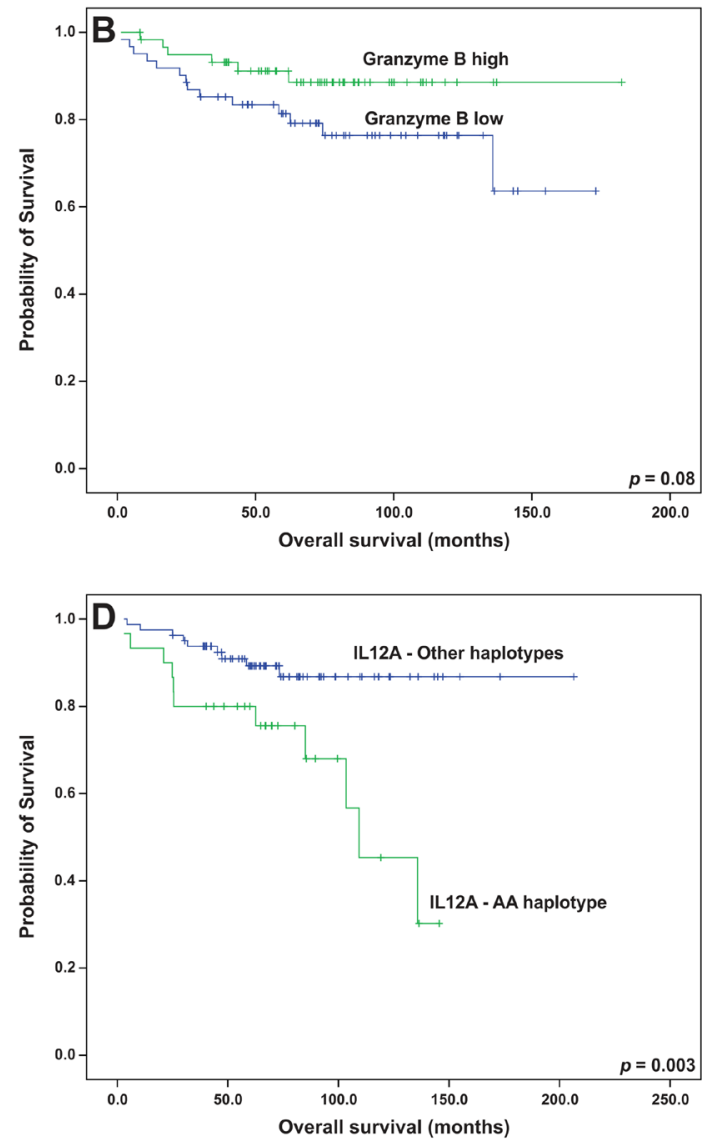

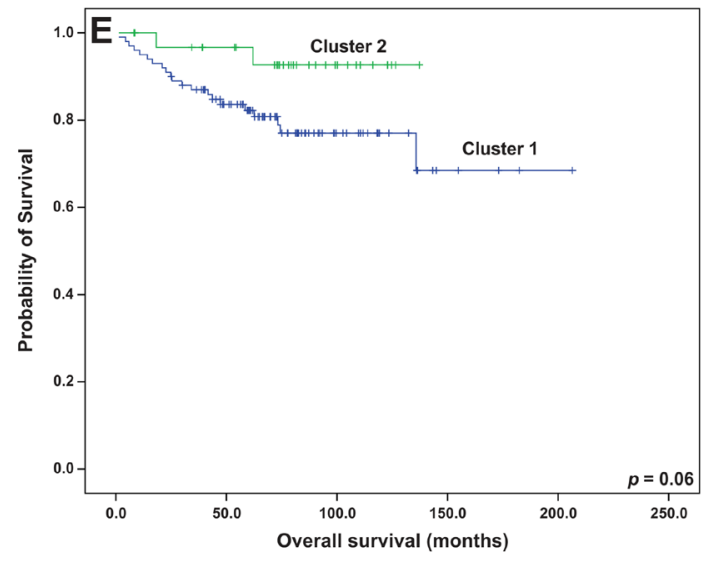

Figure 7: Overall survival analyses (Kaplan-Meier curves) of follicular lymphoma patients treated with R-CHOP/R-CVP influenced by (A) the pattern of FOXP3 cells, (B) intratumoral levels of granzyme B, (C) the genotypes of IL10 rs 1800872 , (D) the presence of the IL12A AA haplotype and (E) microenvironment clusters. All the $p$-values were obtained from log-rank tests. 
It is possible that other SNPs modulate the TME composition without altering the course of FL. For instance, the "A" allele of IL12A rs755004 was associated with increased intratumoral iNOS levels, which may be explained by the role of IL-12A on IFN $\gamma$ secretion, with the consequent recruitment of fagocytes and oxidative burst [31]. We also found that ILI7F rs763780 was associated with granzyme B levels, which may relate to the interference of Th17 cells on cytotoxic lymphocytes [32].

The most significant limitation of our study was its retrospective design. Therefore, the validation of our results using prospective cohorts is warranted. However, several strengths must be recognized. For example, our study had a relatively large sample size, and the patients were homogeneously treated with rituximab for our survival analyses, minimizing any therapy-related biases. We also performed computer-assisted image analyses for the quantification of the IHC markers, which has been proven superior and more reproducible in comparison to simple manual counting [11].

In this study, we demonstrated the adverse prognostic role of several TME elements in FL, considering both protein and genetic data. Importantly, the follicular pattern of the T-CD8+ cells, the high expression of IL-17F (individually and as a cluster member), and the AA haplotype of IL $2 \mathrm{~A}$ were all independently associated with worse prognosis. The latter variable was also associated with the pattern of FOXP3 + cell infiltration, which was validated in this study as a prognostic factor in the rituximab era. We believe that the simultaneous study of the tumor biopsies and immune response related SNPs in patients' peripheral blood allowed for a novel and more integrative approach that will provide new insights on follicular lymphoma biology.

\section{MATERIALS AND METHODS}

\section{Patients, controls and clinical data}

The present retrospective study included an initial number of 237 cases, diagnosed with systemic FL (grades 1, 2, or 3A) between 1999 and 2016 at the Hematology and Hemotherapy Center - UNICAMP and A. C. Camargo Cancer Center.

The clinical data were collected from the medical files. FLIPI was used as the reference prognostic tool.

The study was approved by the Ethics Committees of both institutions (number 32177014.3.0000.5404), and all procedures were carried out according to the Declaration of Helsinki.

\section{SNP selection and genotyping}

The SNPs were studied using a "candidate gene" approach. SNPs located in immune response genes from
A

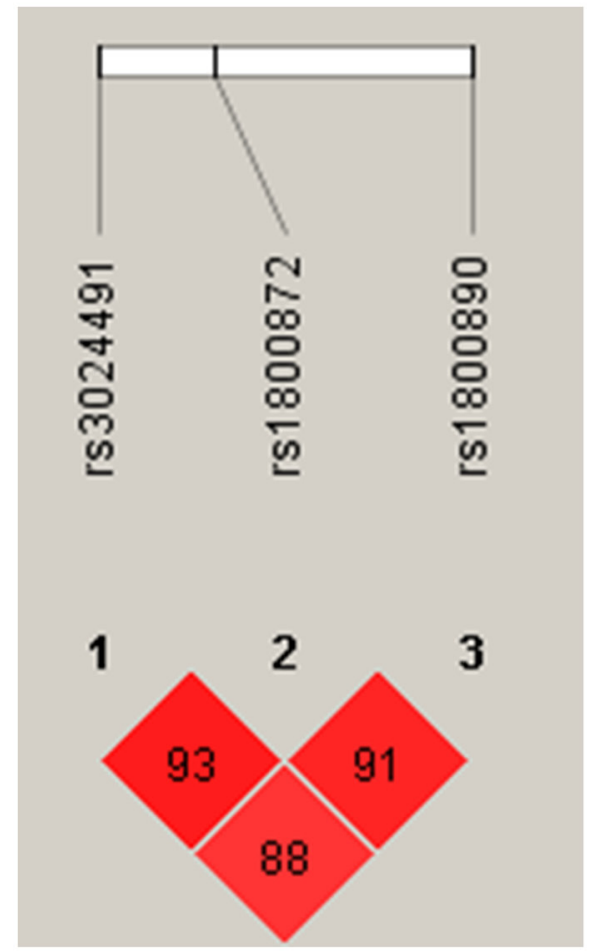

B

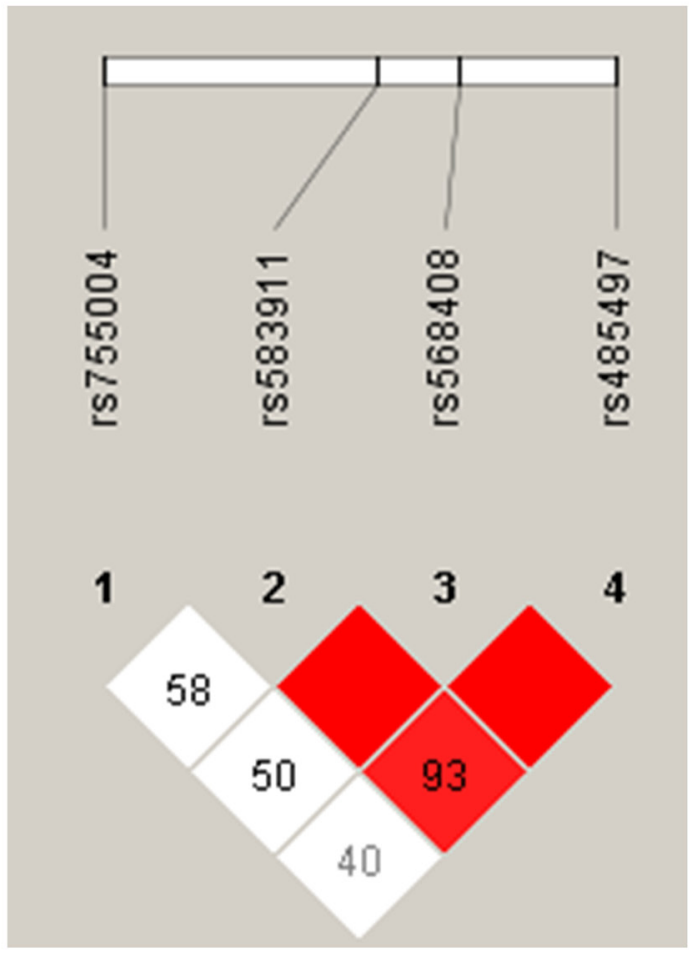

Figure 8: Haplotype estimates in follicular lymphoma patients for (A) chromosome 1 (IL10) and (B) chromosome 3 (IL12A). In each square, the linkage disequilibrium (LD) was estimated between the groups of single nucleotide polymorphisms. The higher LD values (expressed as D') are shown in the red squares. 
Table 8: Single nucleotide polymorphisms (SNPs) assessed in this study

\begin{tabular}{|c|c|c|c|}
\hline Gene & SNP & Previous associations" & References" $^{*}$ \\
\hline IL12A & rs 755004 & Risk for NHL & {$[19]$} \\
\hline IL12A & rs583911 & Risk for ALL & {$[33]$} \\
\hline IL12A & rs568408 & Risk for FL; alterations in $I L 12 \mathrm{~A}$ splicing sites & {$[29,34]$} \\
\hline IL12A & rs485497 & Risk for FL; modulation of IL-12A serum levels & {$[35,36]$} \\
\hline IL 2 & rs2069762 & $\begin{array}{l}\text { Risk for NHL; prognostic factor in FL patients (pre- } \\
\text { rituximab era) }\end{array}$ & {$[13,37]$} \\
\hline$I L 2$ & rs6822844 & Association with multiple autoimmune diseases & [38] \\
\hline ILI0 & rs3024491 & Modulation of IL-10 production & [39] \\
\hline IL10 & rs 1800872 & Modulation of IL-10 production & [40] \\
\hline IL10 & rs 1800890 & Modulation of IL-10 production & [41] \\
\hline TGFB1 & rs6957 & Alteration of macrophage balance and fibrosis & [42] \\
\hline TGFB1 & rs1800469 & $\begin{array}{l}\text { Modulation of TGF } \beta \text { production; prognostic factor in } \\
\text { lung cancer patients }\end{array}$ & {$[43,44]$} \\
\hline$T G F B 1$ & rs 1800471 & $\begin{array}{l}\text { Modulation of TGF } \beta \text { production; association with the } \\
\text { clinical presentation of NHL patients }\end{array}$ & {$[18,45]$} \\
\hline TGFBR1 & rs334348 & $\begin{array}{l}\text { Risk for breast cancer; alteration of specific miRNA } \\
\text { binding sites within } T G F B R 1 \text { gene }\end{array}$ & [46] \\
\hline$T G F B R 2$ & rs3087465 & $\begin{array}{l}\text { Alteration of TGFBR } 2 \text { promoter activity; prognostic } \\
\text { factor in thyroid papillary carcinoma }\end{array}$ & {$[47,48]$} \\
\hline IL17A & rs3748067 & $\begin{array}{l}\text { Risk for gastric cancer; association with breast cancer } \\
\text { molecular subtype and prognosis }\end{array}$ & {$[49,50]$} \\
\hline IL17F & rs763780 & Alteration of IL-17F downstream pathways & [51] \\
\hline
\end{tabular}

(*) References addressing previous associations with cancer susceptibility, progression or with functional alterations of the respective gene/protein. $\mathrm{NHL}=$ non-Hodgkin lymphoma; $\mathrm{FL}=$ follicular lymphoma; $\mathrm{ALL}$ = acute lymphoid leukemia.

the Th1, Th2, or Th17 axis, with previous evidence of active roles in lymphoma, cancer or immune response, were selected. A minor allele frequency of $5 \%$ was required. Sixteen SNPs were then selected, based on previous association studies (Table 8) [13, 18, 19, 29, 33-51].

\section{DNA extraction and genotyping}

DNA was extracted from the peripheral blood of patients and controls using the lithium chloride technique [52]. The DNA yield (ng/uL) and purity (260/280 and 260/230 ratios) assessments were performed using a spectrophotometer (NanoDrop ${ }^{\circledR}$ 2000, ThermoFisher Scientific). The final concentration of all samples was set to $50 \mathrm{ng} / \mathrm{uL}$.

SNP genotyping was performed on the Taqman ${ }^{\circledR}$ OpenArray ${ }^{\circledR}$ System (which is based on quantitative PCR reactions). Briefly, the DNA samples were pipetted together with Taqman $^{\circledR}$ Openarray $^{\circledR}$ Master Mix on 384-well plates. The mixture was transferred to genotyping plates by the Openarray ${ }^{\circledR}$ Accufill $^{\mathrm{TM}}$ system. Thermocycling was performed over 40 cycles, and the visualization of the polymorphic alleles was achieved by VIC ${ }^{\mathrm{TM}}$ and $\mathrm{FAM}^{\mathrm{TM}}$ fluorophores. A single reaction allowed for the simultaneous detection of all 16 polymorphic variants.

\section{Immunohistochemistry}

The immunohistochemical reactions were performed using TMA slides to address the TME composition. A broad panel of TME markers was selected, which encompassed subpopulations of TILs, macrophages and FDCs (CD3, CD4, CD8, CD57, FOXP3, PD1, granzyme $\mathrm{B}$, perforin, CD23, CD68, CD163, iNOS). In addition, for each gene in the SNP approach, the respective protein was detected by immunohistochemistry (IL-12A, IL-2, IL10, TGF $\beta$, TGFBR1, TGFBR2, IL-17A, IL-17F). Details on the clones and dilutions of each antibody are listed in Supplementary Table 5.

The TMA slides were subjected to antigen retrieval using citric acid/pH 6.0 or EDTA/pH 9.0 buffers. Endogenous peroxidase activity was blocked using a $3 \%$ hydrogen peroxide aqueous solution for 20 minutes. The antigen-antibody reaction was performed overnight at $4^{\circ} \mathrm{C}$ and was then amplified using a third-generation polymer that was tagged with anti-mouse and anti-rabbit immunoglobulins and horseradish peroxidase (HRP; Novolink Polymer Detection System, Leica Biosystems, Newcastle Upon Tyne, UK). The reactions were developed with the 3-3'-diaminobenzidine (DAB) chromogenic substrate (Sigma, D5637, St. Louis, MO, USA). Hence, 
the positive cell structures (membrane, cytoplasm or nuclei) presented as a brown color. Positive and negative controls were run for each batch. The negative control was obtained by omitting only the primary antibody used for the reaction.

IHC stain quantification was done automatically using the Aperio Scanscope XT device and by averaging both of the TMA core scores. The Positive Pixel Count algorithm was used to grade the pixels as negative $(\mathrm{N})$, low positive (LP), positive (P) and high positive (HP) (Figure 1). The inputs for the algorithm were a hue value of 0.1 , a hue width of 0.5 and a color saturation threshold of 0.1 (for most cores). In cases with nonspecific background, the color saturation threshold was increased to 0.15 to minimize false-positive detection. For the antibodies that stained specific TME populations (e.g. CD68, FOXP3, CD3), the fraction of all the positive pixels was considered as the score (Score $=\frac{L P+P+H P}{L P+P+H P+N}$ ). For the antibodies that heterogeneously stained both the tumor and microenvironment cells (all the cytokine antibodies), we calculated the H-score [53] using the following formula:

$$
\mathrm{HSCORE}=3\left(\frac{H P}{L P+P+H P+N}\right)+2\left(\frac{P}{L P+P+H P+N)}\right)+1\left(\frac{L P}{L P+P+H P+N)}\right)
$$

For the TIL subpopulations, we also performed "qualitative analyses" by describing the predominant pattern of proliferation $[5,7,8]$. In this setting, there were 4 possible patterns as follows: "intrafollicular" (TILs homogeneously localized inside neoplastic follicles); "perifollicular" (TILs concentrated in the periphery of neoplastic follicles); "interfollicular" (TILs more visualized in the interfollicular spaces); and "diffuse" (TILs well distributed randomly inside and outside the follicles). For statistical purposes, "intrafollicular" and "perifollicular" were both considered "follicular" patterns, whereas "interfollicular" and "diffuse" were reclassified "non-follicular."

For the CD23 stains, we used a "pattern" approach. Thus, the FDC categories were as follows: absent (Group 1); a minority of neoplastic follicles with disrupted meshworks (Group 2); the majority of neoplastic follicles with well- developed meshworks (Group 3); and uniformly well-developed meshworks (Group 4) [54].

\section{Statistical analyses}

We performed the chi-squared $\left(\chi^{2}\right)$, Fisher's exact test, Mann Whitney's test, and Spearman's correlation index to assess the bivariate associations. When necessary, numerical variables were dichotomized as "high" and "low," based on the median levels.

In the SNP study, we tested, for each variant, the HWE, using the $\chi^{2}$ goodness-of-fit test. Pairwise LD analyses were performed to estimate the haplotype formation using Haploview 4.2 (https://www.broadinstitute. org/haploview/haploview). LD was measured by the disequilibrium coefficient $\left(\mathrm{D}^{\prime}\right)$, and LD significance was considered at a $\mathrm{D}^{\prime} \geq 80 \%$.

An exploratory PCA with Varimax rotation was used to address the interactions among the IHC scores. The PCA included both the numerical and categorical variables (pixel counting and patterns of tumor-infiltrating lymphocytes, respectively).

Agglomerative, unsupervised hierarchical clustering was also performed using the numerical IHC data (pixel counting) and using Euclidean distances and Ward's clustering algorithm.

Survival analyses were performed only for the patients that received R-CHOP or R-CVP as a frontline therapy. The OS was defined as the time from diagnosis until death from any cause or the last follow-up. The EFS was defined as the time from diagnosis until death from disease, disease progression or the last followup. The survival curves were plotted using the KaplanMeier method and were compared using a log-rank test. We further performed Cox univariate regressions for the variables that influenced survival based on the KaplanMeier curves. Finally, a Cox multivariate model was proposed, including variables with a $p$ value $\leq 0.10$ in the univariate analysis. The genotyping and IHC data were evaluated separately in the survival models. The prognostic roles of both the PCA and clusters were also examined.

A final $p$ value $<0.05$ was considered statistically significant. When necessary, corrections for multiple comparisons were performed using the BenjaminiHochberg method.

A CONSORT diagram (Supplementary Figure 3) summarizes the workflow of the study, as well as the number of patients for both the IHC and SNP analyses.

\section{Author contributions}

Guilherme Assis Mendonça: designed the project, performed experiments, analyzed the data and wrote the manuscript. André Fattori: analyzed the data and wrote the manuscript. Rafael Rocha: analyzed the data and wrote the manuscript. Gustavo Lourenço: analyzed the data and wrote the manuscript. Márcia Torresan Delamain: analyzed the data. Suely Nonogaki: performed experiments and wrote the manuscript. Vladmir Lima: analyzed the data and wrote the manuscript. Gisele Colleoni: analyzed the data and wrote the manuscript. Cármino Souza: analyzed the data and wrote the manuscript. Fernando Soares: analyzed the data and wrote the manuscript. Carmen Lima: designed the project, analyzed the data and wrote the manuscript. José Vassallo: designed the project, analyzed the data and wrote the manuscript.

\section{ACKNOWLEDGMENTS}

We thank Érika Nascimento, Karina Ramos and Bruna Murbach for the help in collecting patients' blood 
samples. We are also acknowledged to Carlos Nascimento and Severino Ferreira for the help with histological processing.

\section{CONFLICTS OF INTEREST}

Authors have no conflicts of interest to declare.

\section{FUNDING}

This work was funded by Fundação de Amparo à Pesquisa do Estado de São Paulo (FAPESP, research grant \#2014/09854-5) and Coordenação de Aperfeiçoamento de Pessoal de Nível Superior (CAPES, grant \#62/2014).

\section{REFERENCES}

1. Swerdlow SH, Campo E, Pileri SA, Harris NL, Stein H, Siebert R, Advani R, Ghielmini M, Salles GA, Zelenetz AD, Jaffe ES. The 2016 revision of the World Health Organization classification of lymphoid neoplasms. Blood. 2016; 127:2375-2390. https://doi.org/10.1182/ blood-2016-01-643569. [PubMed]

2. Johnson PW, Watt SM, Betts DR, Davies D, Jordan S, Norton AJ, Lister TA. Isolated follicular lymphoma cells are resistant to apoptosis and can be grown in vitro in the CD40/stromal cell system. Blood. 1993; 82:1848-1857. https://doi.org/10.1182/blood.V82.6.1848.1848. [PubMed]

3. Farinha P, Masoudi H, Skinnider BF, Shumansky K, Spinelli JJ, Gill K, Klasa R, Voss N, Connors JM, Gascoyne RD. Analysis of multiple biomarkers shows that lymphoma-associated macrophage (LAM) content is an independent predictor of survival in follicular lymphoma (FL). Blood. 2005; 106:2169-2174. https://doi.org/10.1182/ blood-2005-04-1565. [PubMed]

4. Carreras J, Lopez-Guillermo A, Roncador G, Villamor N, Colomo L, Martinez A, Hamoudi R, Howat WJ, Montserrat E, Campo E. High numbers of tumor-infiltrating programmed cell death 1-positive regulatory lymphocytes are associated with improved overall survival in follicular lymphoma. J Clin Oncol. 2009; 27:1470-1476. https://doi. org/10.1200/JCO.2008.18.0513. [PubMed]

5. Farinha P, Al-Tourah A, Gill K, Klasa R, Connors JM, Gascoyne RD. The architectural pattern of FOXP3positive $\mathrm{T}$ cells in follicular lymphoma is an independent predictor of survival and histologic transformation. Blood. 2010; 115:289-295. https://doi.org/10.1182/ blood-2009-07-235598. [PubMed]

6. Labidi SI, Ménétrier-Caux C, Chabaud S, Chassagne C, Sebban C, Gargi T, Biron P, Blay JY, Ghesquières H. Serum cytokines in follicular lymphoma. Correlation of TGF- $\beta$ and VEGF with survival. Ann Hematol. 2010; 89:25-33. https:// doi.org/10.1007/s00277-009-0777-8. [PubMed]

7. Wahlin BE, Aggarwal M, Montes-Moreno S, Gonzalez LF, Roncador G, Sanchez-Verde L, Christensson B, Sander B,
Kimby E. A unifying microenvironment model in follicular lymphoma: outcome is predicted by programmed death1-positive, regulatory, cytotoxic, and helper $\mathrm{T}$ cells and macrophages. Clin Cancer Res. 2010; 16:637-650. https:// doi.org/10.1158/1078-0432.CCR-09-2487. [PubMed]

8. Smeltzer JP, Jones JM, Ziesmer SC, Grote DM, Xiu B, Ristow KM, Yang ZZ, Nowakowski GS, Feldman AL, Cerhan JR, Novak AJ, Ansell SM. Pattern of CD14+ follicular dendritic cells and PD1+ T cells independently predicts time to transformation in follicular lymphoma. Clin Cancer Res. 2014; 20:2862-2872. https://doi. org/10.1158/1078-0432.CCR-13-2367. [PubMed]

9. Kridel R, Xerri L, Gelas-Dore B, Tan K, Feugier P, Vawda A, Canioni D, Farinha P, Boussetta S, Moccia AA, Brice P, Chavez EA, Kyle AH, et al. The Prognostic Impact of CD163-Positive Macrophages in Follicular Lymphoma: A Study from the BC Cancer Agency and the Lymphoma Study Association. Clin Cancer Res. 2015; 21:34283435. https://doi.org/10.1158/1078-0432.CCR-14-3253. [PubMed]

10. de Jong D, Koster A, Hagenbeek A, Raemaekers J, Veldhuizen D, Heisterkamp S, de Boer JP, van Glabbeke $\mathrm{M}$. Impact of the tumor microenvironment on prognosis in follicular lymphoma is dependent on specific treatment protocols. Haematologica. 2009; 94:70-77. https://doi. org/10.3324/haematol.13574. [PubMed]

11. Sander B, de Jong D, Rosenwald A, Xie W, Balagué O, Calaminici M, Carreras J, Gaulard P, Gribben J, Hagenbeek A, Kersten MJ, Molina TJ, Lee A, et al. The reliability of immunohistochemical analysis of the tumor microenvironment in follicular lymphoma: a validation study from the Lunenburg Lymphoma Biomarker Consortium. Haematologica. 2014; 99:715-725. https://doi. org/10.3324/haematol.2013.095257. [PubMed]

12. Rothman N, Skibola CF, Wang SS, Morgan G, Lan Q, Smith MT, Spinelli JJ, Willett E, De Sanjose S, Cocco P, Berndt SI, Brennan P, Brooks-Wilson A, et al. Genetic variation in TNF and IL10 and risk of non-Hodgkin lymphoma: a report from the InterLymph Consortium. Lancet Oncol. 2006; 7:27-38. https://doi.org/10.1016/S1470-2045(05)70434-4. [PubMed]

13. Cerhan JR, Wang S, Maurer MJ, Ansell SM, Geyer SM, Cozen W, Morton LM, Davis S, Severson RK, Rothman N, Lynch CF, Wacholder S, Chanock SJ, et al. Prognostic significance of host immune gene polymorphisms in follicular lymphoma survival. Blood. 2007; 109:5439-5446. https://doi.org/10.1182/blood-2006-11-058040. [PubMed]

14. Krejsgaard T, Litvinov IV, Wang Y, Xia L, WillerslevOlsen A, Koralov SB, Kopp KL, Bonefeld CM, Wasik MA, Geisler C, Woetmann A, Zhou Y, Sasseville D, Odum N. Elucidating the role of interleukin- $17 \mathrm{~F}$ in cutaneous T-cell lymphoma. Blood. 2013; 122:943-950. https://doi. org/10.1182/blood-2013-01-480889. [PubMed]

15. Lu T, Yu S, Liu Y, Yin C, Ye J, Liu Z, Ma D, Ji C. Aberrant Circulating Th17 Cells in Patients with B-Cell Non- 
Hodgkin's Lymphoma. PLoS One. 2016; 11:e0148044. https://doi.org/10.1371/journal.pone.0148044. [PubMed]

16. Chen X, Han S, Wang S, Zhou X, Zhang M, Dong J, Shi X, Qian N, Wang X, Wei Q, Shen H, Hu Z. Interactions of $I L-12 A$ and $I L-12 B$ polymorphisms on the risk of cervical cancer in Chinese women. Clin Cancer Res. 2009; 15:400 405. https://doi.org/10.1158/1078-0432.CCR-08-1829. [PubMed]

17. Yang ZZ, Grote DM, Ziesmer SC, Niki T, Hirashima M, Novak AJ, Witzig TE, Ansell SM. IL-12 upregulates TIM-3 expression and induces $\mathrm{T}$ cell exhaustion in patients with follicular B cell non-Hodgkin lymphoma. J Clin Invest. 2012; 122:1271-1282. https://doi.org/10.1172/JCI59806. [PubMed]

18. Mazur G, Bogunia-Kubik K, Wrobel T, Kuliczkowski K, Lange A. TGF-beta1 gene polymorphisms influence the course of the disease in non-Hodgkin's lymphoma patients. Cytokine. 2006; 33:145-149. https://doi.org/10.1016/j. cyto.2005.12.010. [PubMed]

19. Butterbach K, Beckmann L, de Sanjosé S, Benavente Y, Becker N, Foretova L, Maynadie M, Cocco P, Staines A, Boffetta P, Brennan P, Nieters A. Association of JAK-STAT pathway related genes with lymphoma risk: results of a European case-control study (EpiLymph). Br J Haematol. 2011; 153:318-333. $\quad$ https://doi.org/10.1111/j.13652141.2011.08632.x. [PubMed]

20. Menter T, Tzankov A, Zucca E, Kimby E, Hultdin M, Sundström C, Beiske K, Cogliatti S, Banz Y, Cathomas G, Karjalainen-Lindsberg ML, Grobholz R, Mazzucchelli L, et al. Prognostic implications of the microenvironment for follicular lymphoma under immunomodulation therapy. $\mathrm{Br}$ J Haematol. 2020; 189:707-717. https://doi.org/10.1111/ bjh.16414. [PubMed]

21. Hilchey SP, De A, Rimsza LM, Bankert RB, Bernstein SH. Follicular lymphoma intratumoral CD4+CD25+GITR+ regulatory $\mathrm{T}$ cells potently suppress CD3/CD28costimulated autologous and allogeneic CD8+CD25- and CD4+CD25- T cells. J Immunol. 2007; 178:4051-4061. https://doi.org/10.4049/jimmunol.178.7.4051. [PubMed]

22. Amé-Thomas $\mathrm{P}$, Tarte $\mathrm{K}$. The yin and the yang of follicular lymphoma cell niches: role of microenvironment heterogeneity and plasticity. Semin Cancer Biol. 2014; 24:23-32. https://doi.org/10.1016/j.semcancer.2013.08.001. [PubMed]

23. Behr FM, Chuwonpad A, Stark R, van Gisbergen KP. Armed and Ready: Transcriptional Regulation of Tissue-Resident Memory CD8 T Cells. Front Immunol. 2018; 9:1770. https:// doi.org/10.3389/fimmu.2018.01770. [PubMed]

24. Laurent C, Müller S, Do C, Al-Saati T, Allart S, Larocca LM, Hohaus S, Duchez S, Quillet-Mary A, Laurent G, Brousset P, Valitutti S. Distribution, function, and prognostic value of cytotoxic T lymphocytes in follicular lymphoma: a 3-D tissue-imaging study. Blood. 2011; 118:5371-5379. https://doi.org/10.1182/blood-2011-04-345777. [PubMed]
25. Ramsay AG, Clear AJ, Kelly G, Fatah R, Matthews J, Macdougall F, Lister TA, Lee AM, Calaminici M, Gribben JG. Follicular lymphoma cells induce T-cell immunologic synapse dysfunction that can be repaired with lenalidomide: implications for the tumor microenvironment and immunotherapy. Blood. 2009; 114:4713-4720. https://doi.org/10.1182/ blood-2009-04-217687. [PubMed]

26. Kiaii S, Clear AJ, Ramsay AG, Davies D, Sangaralingam A, Lee A, Calaminici M, Neuberg DS, Gribben JG. Follicular lymphoma cells induce changes in T-cell gene expression and function: potential impact on survival and risk of transformation. J Clin Oncol. 2013; 31:2654-2661. https:// doi.org/10.1200/JCO.2012.44.2137. [PubMed]

27. Ochando J, Braza MS. T follicular helper cells: a potential therapeutic target in follicular lymphoma. Oncotarget. 2017; 8:112116-112131. https://doi.org/10.18632/oncotarget.22788. [PubMed]

28. Aschebrook-Kilfoy B, Zheng T, Foss F, Ma S, Han X, Lan Q, Holford T, Chen Y, Leaderer B, Rothman N, Zhang Y. Polymorphisms in immune function genes and nonHodgkin lymphoma survival. J Cancer Surviv. 2012; 6:102114. https://doi.org/10.1007/s11764-010-0164-4. [PubMed]

29. Liu L, Xu Y, Liu Z, Chen J, Zhang Y, Zhu J, Liu J, Liu S, Ji G, Shi H, Shen H, Hu Z. IL12 polymorphisms, HBV infection and risk of hepatocellular carcinoma in a high-risk Chinese population. Int J Cancer. 2011; 128:1692-1696. https://doi.org/10.1002/ijc.25488. [PubMed]

30. Zhao J, Zhao J, Perlman S. Differential effects of IL-12 on Tregs and non-Treg T cells: roles of IFN- $\gamma$, IL-2 and IL-2R. PLoS One. 2012; 7:e46241. https://doi.org/10.1371/journal. pone.0046241. [PubMed]

31. Trinchieri G, Gerosa F. Immunoregulation by interleukin-12. J Leukoc Biol. 1996; 59:505-511. https:// doi.org/10.1002/j1b.59.4.505. [PubMed]

32. Duan MC, Huang Y, Zhong XN, Tang HJ. Th17 cell enhances CD8 T-cell cytotoxicity via IL-21 production in emphysema mice. Mediators Inflamm. 2012; 2012:898053. https://doi.org/10.1155/2012/898053. [PubMed]

33. Chang JS, Wiemels JL, Chokkalingam AP, Metayer C, Barcellos LF, Hansen HM, Aldrich MC, Guha N, Urayama KY, Scélo G, Green J, May SL, Kiley VA, et al. Genetic polymorphisms in adaptive immunity genes and childhood acute lymphoblastic leukemia. Cancer Epidemiol Biomarkers Prev. 2010; 19:2152-2163. https:// doi.org/10.1158/1055-9965.EPI-10-0389. [PubMed]

34. Chen Y, Zheng T, Lan Q, Foss F, Kim C, Chen X, Dai M, Li Y, Holford T, Leaderer B, Boyle P, Chanock SJ, Rothman N, Zhang Y. Cytokine polymorphisms in Th1/ Th2 pathway genes, body mass index, and risk of nonHodgkin lymphoma. Blood. 2011; 117:585-590. https:// doi.org/10.1182/blood-2010-07-295097. [PubMed]

35. Lan Q, Wang SS, Menashe I, Armstrong B, Zhang Y, Hartge P, Purdue MP, Holford TR, Morton LM, Kricker A, Cerhan 
JR, Grulich A, Cozen W, et al. Genetic variation in Th1/ Th2 pathway genes and risk of non-Hodgkin lymphoma: a pooled analysis of three population-based case-control studies. Br J Haematol. 2011; 15:341-350. https://doi. org/10.1111/j.1365-2141.2010.08424.x. [PubMed]

36. Fogel O, Rivière E, Seror R, Nocturne G, Boudaoud S, Ly B, Gottenberg JE, Le Guern V, Dubost JJ, Nititham J, Taylor KE, Chanson P, Dieudé P, et al. Role of the IL-12/IL-35 balance in patients with Sjögren syndrome. J Allergy Clin Immunol. 2018; 142:258-268.e5. https://doi.org/10.1016/j. jaci.2017.07.041. [PubMed]

37. Song $\mathrm{H}$, Chen L, Cha Z, Bai J. Interleukin 2 gene polymorphisms are associated with non-Hodgkin lymphoma. DNA Cell Biol. 2012; 31:1279-1284. https:// doi.org/10.1089/dna.2011.1603. [PubMed]

38. Maiti AK, Kim-Howard X, Viswanathan P, Guillén L, Rojas-Villarraga A, Deshmukh H, Direskeneli $\mathrm{H}$, Saruhan-Direskeneli G, Cañas C, Tobön GJ, Sawalha AH, Cherñavsky AC, Anaya JM, Nath SK. Confirmation of an association between rs6822844 at the I12-I121 region and multiple autoimmune diseases: evidence of a general susceptibility locus. Arthritis Rheum. 2010; 62:323-329. https://doi.org/10.1002/art.27222. [PubMed]

39. Assis S, Marques CR, Silva TM, Costa RS, AlcantaraNeves NM, Barreto ML, Barnes KC, Figueiredo CA. IL10 single nucleotide polymorphisms are related to upregulation of constitutive IL-10 production and susceptibility to Helicobacter pylori infection. Helicobacter. 2014; 19:168173. https://doi.org/10.1111/hel.12119. [PubMed]

40. Lowe PR, Galley HF, Abdel-Fattah A, Webster NR. Influence of interleukin-10 polymorphisms on interleukin-10 expression and survival in critically ill patients. Crit Care Med. 2003; 31:34-38. https://doi. org/10.1097/00003246-200301000-00005. [PubMed]

41. Gibson AW, Edberg JC, Wu J, Westendorp RG, Huizinga TW, Kimberly RP. Novel single nucleotide polymorphisms in the distal IL-10 promoter affect IL-10 production and enhance the risk of systemic lupus erythematosus. J Immunol. 2001; 166:3915-3922. https://doi.org/10.4049/ jimmunol.166.6.3915. [PubMed]

42. Ierodiakonou D, Postma DS, Koppelman GH, Gerritsen J, ten Hacken NH, Timens W, Boezen HM, Vonk JM. TGF- $\beta 1$ polymorphisms and asthma severity, airway inflammation, and remodeling. J Allergy Clin Immunol. 2013; 131:582585. https://doi.org/10.1016/j.jaci.2012.08.013. [PubMed]

43. Grainger DJ, Heathcote K, Chiano M, Snieder H, Kemp PR, Metcalfe JC, Carter ND, Spector TD. Genetic control of the circulating concentration of transforming growth factor type $\beta 1$. Hum Mol Genet. 1999; 8:93-97. https://doi. org/10.1093/hmg/8.1.93. [PubMed]

44. Yuan X, Wei Q, Komaki R, Liu Z, Yang J, Tucker SL, Xu T, Heymach JV, Lu C, Cox JD, Liao Z. TGF $\beta 1$ Polymorphisms Predict Distant Metastasis-Free Survival in Patients with
Inoperable Non-Small-Cell Lung Cancer after Definitive Radiotherapy. PLoS One. 2013; 8:e65659. https://doi. org/10.1371/journal.pone.0065659. [PubMed]

45. Awad MR, El-Gamel A, Hasleton P, Turner DM, Sinnott PJ, Hutchinson IV. Genotypic variation in the transforming growth factor- $\beta 1$ gene: association with transforming growth factor- $\beta 1$ production, fibrotic lung disease, and graft fibrosis after lung transplantation. Transplantation. 1998; 66:1014-1020. https://doi.org/10.1097/00007890199810270-00009. [PubMed]

46. Nicoloso MS, Sun H, Spizzo R, Kim H, Wickramasinghe P, Shimizu M, Wojcik SE, Ferdin J, Kunej T, Xiao L, Manoukian S, Secreto G, Ravagnani F, et al. Singlenucleotide polymorphisms inside microRNA target sites influence tumor susceptibility. Cancer Res. 2010; 70:27892798. https://doi.org/10.1158/0008-5472.CAN-09-3541. [PubMed]

47. Seijo ER, Song H, Lynch MA, Jennings R, Qong X, Lazaridis E, Muro-Cacho C, Weghorst CM, MuñozAntonia T. Identification of genetic alterations in the TGFbeta type II receptor gene promoter. Mutat Res. 2001; 483:19-26. https://doi.org/10.1016/S00275107(01)00217-2. [PubMed]

48. Choe BK, Kim SK, Park HJ, Park HK, Kwon KH, Lim SH, Yim SV. Polymorphisms of TGFBR2 contribute to the progression of papillary thyroid carcinoma. Mol Cell Toxicol. 2012; 8:1-8. https://doi.org/10.1007/s13273-0120001-0.

49. Wang L, Jiang Y, Zhang Y, Wang Y, Huang S, Wang Z, Tian B, Yang Y, Jiang W, Pang D. Association analysis of IL17A and IL-17F polymorphisms in Chinese Han women with breast cancer. PLoS One. 2012; 7:e34400. https://doi. org/10.1371/journal.pone.0034400. [PubMed]

50. Qinghai Z, Yanying W, Yunfang C, Xukui Z, Xiaoqiao Z. Effect of interleukin-17A and interleukin-17F gene polymorphisms on the risk of gastric cancer in a Chinese population. Gene. 2014; 537:328-332. https://doi. org/10.1016/j.gene.2013.11.007. [PubMed]

51. Kawaguchi M, Takahashi D, Hizawa N, Suzuki S, Matsukura S, Kokubu F, Maeda Y, Fukui Y, Konno S, Huang SK, Nishimura M, Adachi M. IL-17F sequence variant (His161Arg) is associated with protection against asthma and antagonizes wild-type IL-17F activity. J Allergy Clin Immunol. 2006; 117:795-801. https://doi. org/10.1016/j.jaci.2005.12.1346. [PubMed]

52. Woodhead JL, Fallon R, Figueredo H, Landgale J, Malcolm ADB. Alternative methodology of gene diagnosis. In: Davies KE, editor. Human Genetic Diseases: A Practical Approach. Oxford: IRL Press; 1986. pp. 51-64.

53. Byers RJ, Currie T, Tholouli E, Rodig SJ, Kutok JL. MSI2 protein expression predicts unfavorable outcome in acute myeloid leukemia. Blood. 2011; 118:2857-2867. https:// doi.org/10.1182/blood-2011-04-346767. [ubMed] 
54. Glas AM, Knoops L, Delahaye L, Kersten MJ, Kibbelaar RE, Wessels LA, van Laar R, van Krieken JH, Baars JW, Raemaekers J, Kluin PM, van't Veer LJ, de Jong D. Geneexpression and immunohistochemical study of specific T-cell subsets and accessory cell types in the transformation and prognosis of follicular lymphoma. J Clin Oncol. 2007; 25:390-398. https://doi.org/10.1200/JCO.2006.06.1648. [PubMed] 